\title{
Beaming in on some stimulating issues in stellar evolution
}

\author{
A.M.S Richards*, \\ Jodrell Bank Observatory, University of Manchester, SK11 9DL, UK \\ E-mail: amsrejb.man.ac.uk
}

This review discusses some recent maser observations and their interpretation, with the emphasis on physical processes in star formation and mass loss from evolved stars. I draw attention to issues where high-resolution observations have led to new, surprising or controversial developments. I also mention some results and prospects for maser studies arising from new instruments and surveys. Highlights include measurements of Zeeman splitting in non-paramagnetic species such as water and methanol. The water maser results often indicate a much stronger magnetic field than is seen by nearby $\mathrm{OH}$ masers, suggesting that the former arise from overdense clumps. Maser scattering or Faraday rotation implicates a significant ionised fraction. The increasing number of axisymmetric outflows from evolved stars, often with no signs of rotation, are likely to be shaped by magnetic fields, but there is no agreement on their origin. Extraordinary thousand-AU filaments have been seen in e.g. $\mathrm{W} 3(\mathrm{OH})$ in $\mathrm{OH}$ and methanol, and in $\mathrm{S} 128$ in water. At the other extreme, compact 6-GHz $\mathrm{OH}$ and methanol are associated on sub-arcsec, but not milli-arcsec scales; does this indicate chemical chronology or competitive maser amplification? Another surprise is the increasing number of weak methanol and water masers associated with low-mass young stellar objects. Masers are still an ideal tool to probe the chemistry, kinematics, density, magnetic field and ionised fraction, and hence the rate and clumping scales of star formation but the process is intrinsically a lot more complex than it once seemed.

The 8th European VLBI Network Symposium

September 26-29, 2006

Toruń, Poland

${ }^{*}$ Speaker. 

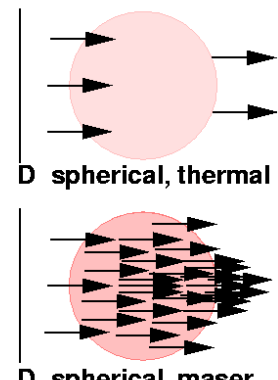

spherical, maser

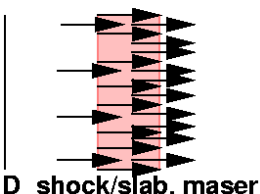

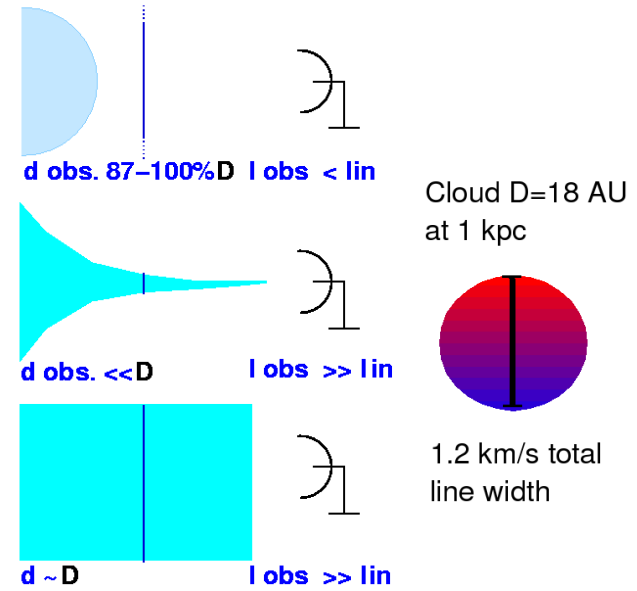
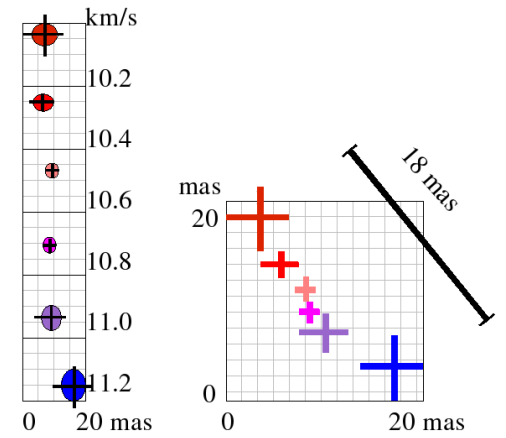

Figure 1: left The top row shows radiation $I_{\text {in }}$ incident on a spherical cloud of moderate optical depth and diameter $D$. The observed intensity $I_{\mathrm{obs}}$ is less than $I_{\text {in }}$ and if the cloud can be spatially resolved the apparent diameter $d$ is typically $\sim 87 \% D$ at full width half maximum (FWHM). The second row shows the same situation for a maser cloud with large negative optical depth. The path length through the centre of the cloud is greater than through the limbs and, as long as the maser is unsaturated, the centre is amplified much more. Not only is $I_{\mathrm{obs}} \gg I_{\mathrm{in}}$, but the FWHM of an individual maser spot is a small fraction of $D$ and is an inverse function of the amplification. The third row shows maser amplification perpendicular to the shock direction in a shocked slab. Amplification is highly efficient but the apparent size of each masing component can remain closer to the actual size of the whole region. right The appearance of a simplified spherical RSG maser cloud with a velocity difference of $1.2 \mathrm{~km} \mathrm{~s}^{-1}$ (a typical thermal line width) over $D$. The middle plot shows a series of interferometer channel maps; each samples emission in a $0.2 \mathrm{~km} \mathrm{~s}^{-1}$ bin and the apparent spot (beamed) FWHM is inversely related to the flux density in the channel. The relative positions of the emission from channel to channel across the cloud show the total unbeamed size of the cloud, plotted on the right.

\section{What are masers?}

This section presents a brief and eclectic introduction to the origins of maser emission associated with the interstellar medium (ISM) and the birth, old age and death of stars. Masers are found in:

- Star-forming regions (SFR):

- clouds of molecules sublimated off grains in the early stages of cloud collapse

- discs and outflows associated with more evolved young stellar objects (YSOs)

- Evolved (post-main-sequence) stars:

- circumstellar envelopes (CSE) around asymptotic giant branch (AGB) stars such as Mira variables and their high-mass analogues, red supergiants (RSG)

- more strongly asymmetric and bipolar outflows from (proto)planetary nebulae, (P)PNe

- Supernova remnants (SNR) 
I only discuss the strongest common $\mathrm{cm}$-wave masers accessible to radio interferometry: $\mathrm{SiO}, \mathrm{H}_{2} \mathrm{O}, \mathrm{OH}$ and $\mathrm{CH}_{3} \mathrm{OH} . \mathrm{H}_{2} \mathrm{CO}$ is less well-studied but has produced some interesting results e.g.[72]. I do not cover extragalactic masers (the subject of another review in these proceedings[106]) nor masers associated with solar system objects such as comets. More complete explanations and reviews are given in $[22,39,93,3,20]$. Most of the discoveries reviewed here involve highresolution observations and there is no space to do justice to the importance of long-term single-dish monitoring using facilities including Nançay (e.g. ground-state $\mathrm{OH}[47]$ ), Effelsberg (e.g. excited $\mathrm{OH}[34])$ or Puschino $\left(\mathrm{H}_{2} \mathrm{O}[85,86]\right)$, nor blind surveys, notably for methanol[43, 122, 23].

\subsection{Conditions for masing}

Microwave Amplification of Stimulated Emitted Radiation (MASER) occurs when a higherenergy-level state is overpopulated with respect to a lower level. The pumping mechanism is generally either collisional or radiative (typically mid- or far-IR emission from dust in the latter case), exciting molecules to a higher state, which then decay ("cascade") until a bottleneck is reached at the upper maser state. One incident photon with a wavelength corresponding to the energy difference between the maser states can trigger the emission of a second photon, then four, and so on, leading to exponential amplification as long as the population inversion is maintained. This is unsaturated emission, shown in Fig. 1 left middle row. If the maser emission rate reaches the pump rate, for example where the path length is longest such as through the centre of a cloud, the maser becomes saturated and amplification is close to linear. The 'seed' photons are usually ambient radiation which (in Galactic sources) is usually undetectable, with rare but interesting exceptions. The probability of stimulated emission is proportional to $\lambda^{4}$, hence the preponderance of longwavelength masers. Masers can reach very high brightness temperatures (insofar as the concept is meaningful at all for such extremely non-thermal emission); $10^{16} \mathrm{~K}$ was recorded during a flare in Orion KL [114]. The masing rate must exceed that of collisional de-excitation.

These constraints allow the physical conditions in masing regions to be modelled. The lowestfrequency masing states detected from $\mathrm{OH}, \mathrm{H}_{2} \mathrm{O}$ and $\mathrm{SiO}$ typically sample temperature $(T)$ ranges of 50-500, 500-1500 and 1500-2500 K, respectively, but the wide variety of masing transitions in each species and less common pump processes often produce surprises. Number densities $(n)$ are usually in the range $10^{12}-10^{16} \mathrm{~m}^{-3}$ and the velocity gradient must not be too great, e.g. not more than a thermal line width over a region deep enough to provide a high enough column density; this varies from $0.1 \mathrm{AU}$ in a hot dense $\mathrm{SiO}$ region, to tens of $\mathrm{AU}$ for $\mathrm{OH} 1612-\mathrm{MHz}$ masers. On the other hand, the locality must be optically thin to the pump cascade photons - but separate regions along the line of sight can provide mutual stimulation or amplification if the velocities are correct (non-local overlap).

Typical maser local environments include dense, dusty, shielded clumps in a CSE within tens of stellar radii of the central star and post-shock gas resulting from the impact of star formation on the ISM. Two separate clumps overlapping along the line of sight can produce dramatic maser amplification $[32,38]$. A shock-compressed slab which is optically thin along the short axis but provides a long amplification path length perpendicular to the shock, provides an especially favourable environment[40] (Fig. 1 left bottom row). The morphology and velocity structure of a masing region can in some cases be measured directly[109] or modelled from maser beaming[130]; an understanding of this is also required to interpret the saturation state and thus the physical conditions. 
Spatial beaming is illustrated in Fig. 1, which also shows how the physical size of a maser cloud can be recovered.

Maser beaming from an asymmetric environment implies that detectability can depend strongly on the viewing angle. This is true not only for the amplification of background emission, but for regions where there is only sufficient velocity-coherent column density along a particular line of sight. If a maser is close to saturation, this is further exacerbated by competitive beaming. Masers from a shock front will only be detectable from directions close to perpendicular to the shock direction and there are profound implications for the interpretation of maser variability; see for example Sections 5.1 and 4.4 .

\subsection{Pulsar proof of masing populations}

Direct observational evidence for the different conditions in masing regions and the effects of competitive gain or non-local overlap between $\mathrm{OH}$ transitions was provided by detection of maser emission from the SFR G339.1-0.4 along the line of sight towards the pulsar PSR B1641-45[137]. The pulsar beam selects a very narrow column, experiencing different conditions from the whole Parkes telescope field of view (10 arcmin) and the stacked on-pulse spectrum shows significant deviations from the off-pulse spectrum, including:

- A brighter, narrower on-pulse feature is seen at $1720 \mathrm{MHz}$;

- The conjugate 1612-MHz absorption shows complementary behaviour;

- 1612-, 1665- and 1667- MHz absorption features at other velocities are deeper on-pulse;

- High-velocity lines seen off-pulse vanish on-pulse;

- Low velocity lines seen in absorption off-pulse appear in emission on-pulse.

\subsection{Masers as astrometric tools}

VLBI of bright masers can achieve $\mu$ arcsec resolution and the increasing ease of phasereferenced VLBI and monitoring is producing many results based on high astrometric accuracy. Measurements of parallax using VERA include a distance for UX Cyg[81] and other reports in these proceedings $[79,75]$. Some of the advances which are possible now that different maser species can be aligned accurately are discussed in Section 3.1.

\section{Masers get everywhere}

The very small size of masers (in both angular and spectral dimensions) means that, until recently, most maser searches involved objects selected by close analogy to the original maser detections (e.g. using IR colours or physical similarity). Faster instruments and data reduction techniques now allow blind single-dish surveys and 'fishing expeditions' in new classes of object. Interferometry follow-up is important to confirm the identifications and to provide enough detail in the maser regions to constrain models of the physical conditions. 


\section{1 $\mathrm{SiO}$ masers}

\subsubsection{Scutum star cluster}

IR observations revealed that a star cluster in Scutum contains 14 RSG[50], the most ever detected in a single cluster. Their evolved nature was reinforced by detection of $\mathrm{SiO}$ maser emission[102]. This also allowed a much more precise determination of the kinematic distance of the cluster (slightly increasing the inferred IR luminosities, thus confirming the supergiant status of the objects) and of the velocity dispersion, constraining the age and mass of the cluster.

\subsubsection{Globular clusters}

Even more surprisingly, $\mathrm{SiO}$ maser emission has been detected towards five globular clusters in the bulge and disc[90]. The presence of $\mathrm{SiO}$ implies that these clusters are approaching $10 \mathrm{~Gy}$ old and solar-mass stars are reaching the AGB. These have a strong enough oxygen-rich wind to support masers, unlike more rapidly-evolving higher-mass stars, under the low-metallicity conditions in globular clusters.

\subsubsection{Post-nova condensation}

$\mathrm{SiO}$ masers were found close to the photosphere of V838 Mon[33], 3 years after its nova-like eruption observed in 2002[99]. This confirms that the system has suddenly produced an AGB-like, cool, mass-losing star and opens up the intriguing possibility that other evolved stars with $\mathrm{SiO}$ masers have similar origins. The maser discovery also allows the kinematic distance to be refined.

\subsection{Carbon star enigma solved}

If optical/IR spectroscopy of an AGB star shows a high proportion of lines from carbon-rich species such as CN (a $\mathrm{C} \star$ ), the CSE, generated by mass-loss from the star, is normally C-rich also. Silicate dust features and $\mathrm{OH}$ or water masers indicate an O-rich wind so it is surprising that these have been detected around some $\mathrm{C} \star \mathrm{s}$ (e.g. [45] and references therein). The contradictory features could arise from separate components of an AGB-AGB binary[87], but the companion $\mathrm{O} \star \mathrm{s}$ have never been detected[44]. Alternatively, an extreme stellar convective event (thermal pulse) could have converted the stellar photospheric composition from O-to C-rich, taking perhaps a decade to propagate through the inner CSE where water masers are found[138]. This is unlikely to be the case for the $\mathrm{C} \star \mathrm{V} 778 \mathrm{Cyg}$ as its silicate features have remained stable for at least 14 years[141], suggesting a third hypothesis: the O-rich wind is left over from the pre-thermal-pulse stage of the $\mathrm{C} \star$ but it is stored in a disc around a lower-mass main sequence companion. The water maser spectrum of V778 Cyg has also been stable since first detected in the 1980s[101].

The water masers were resolved for the first time, using MERLIN, in 2001[118]. Figure 2 shows that the masers have a monotonic velocity gradient, seen more clearly if velocity is plotted against the major axis of the region (Fig. 3 left). The system is at a distance of $\sim 1.4 \mathrm{kpc}[141]$ and we initially modelled the masers as an almost edge-on disc in Keplerian rotation. More recently, a warped-disc model[6] has taken into account the deviations from linearity and the possibility that only part of the disc is seen. These constraints place the companion mass in the range $0.17-$ $1.7 \mathrm{M}_{\odot}$, so the $\mathrm{C} \star$ could easily have had a higher progenitor mass and evolved more rapidly. Such a main-sequence companion would not have been separated from the $\mathrm{C} \star$ by existing optical 


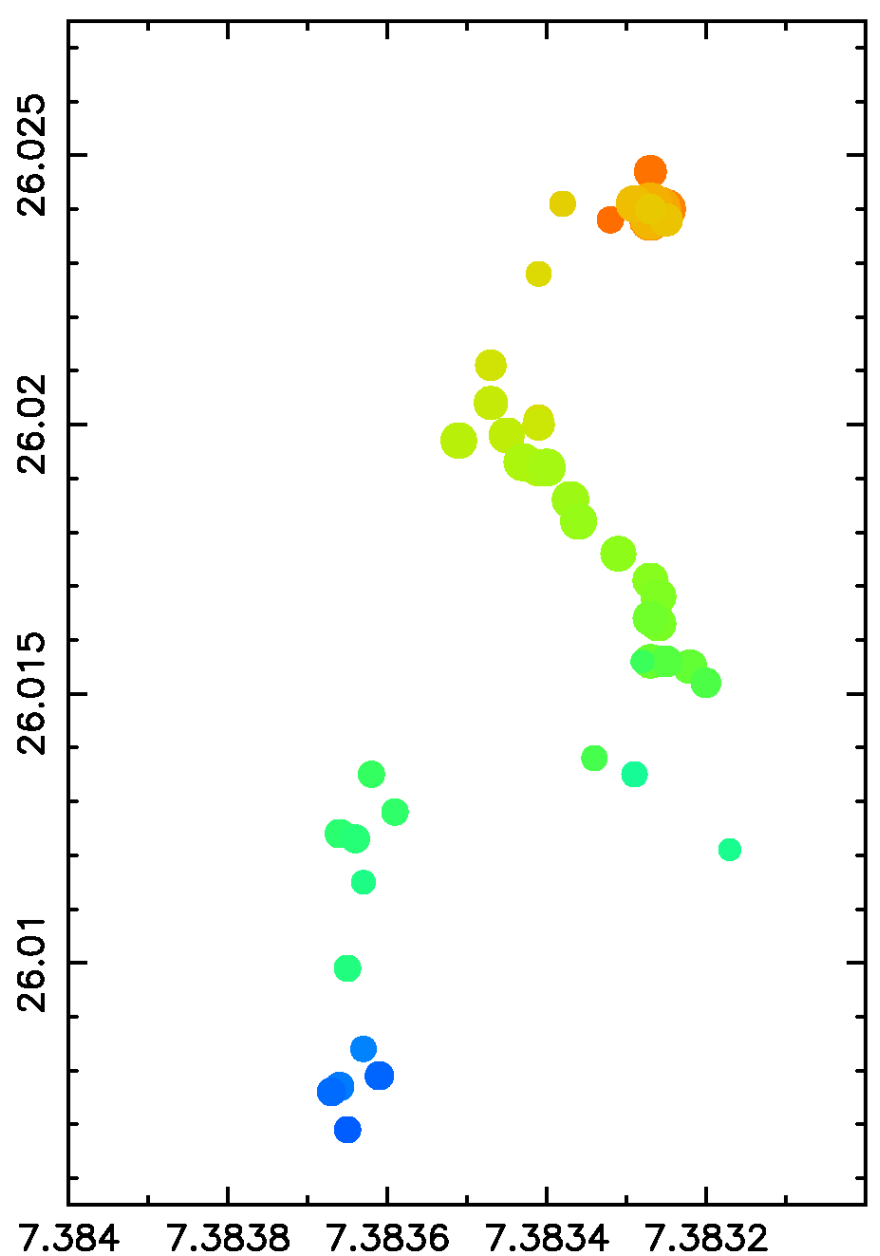

Figure 2: The distribution of water masers around V778 Cyg, coloured according to relative velocity and scaled according to flux density. The $x$ and $y$ positions are in seconds and arcseconds offsets from right ascension $20^{\mathrm{h}} 36^{\mathrm{m}}+60^{\circ} 05^{\prime}$, respectively.

observations, especially in a dusty environment. The maser images are phase-referenced giving a position accuracy of the centroid of $\leq 25$ mas. The most likely position of the $\mathrm{C} \star$ (taking proper motion into account) is at a separation of 192 mas; Fig. 3 right shows the allowed (shaded) region. The maser features have changed in velocity by $<0.5 \mathrm{~km} \mathrm{~s}^{-1}$ in 15 years, also consistent with the orbital velocity at a separation of this magnitude.

The observed disc kinematics and IR measurements[141] were used to deduce the disc mass ( $\left.2 \times 10^{-6} \mathrm{M}_{\odot}\right), T \sim 300 \mathrm{~K}$, thickness and optical depth, and thus to predict the maser characteristics. The model results are closely consistent with the observed brightness temperatures. The model allows the dust grains to be relatively large $(1 \mu \mathrm{m})$, consistent with grain growth in a disc which has been stable for many decades, at a lower $T$ (due to the greater distance from the AGB star) than the normal water maser zone around AGB stars. The enigma of a $\mathrm{C} \star$ with silicate features and water masers has been solved for V778 Cyg as these results demonstrate that the mass lost during an earlier $\mathrm{O} \star$ phase has accumulated in a reservoir around a main-sequence companion. 

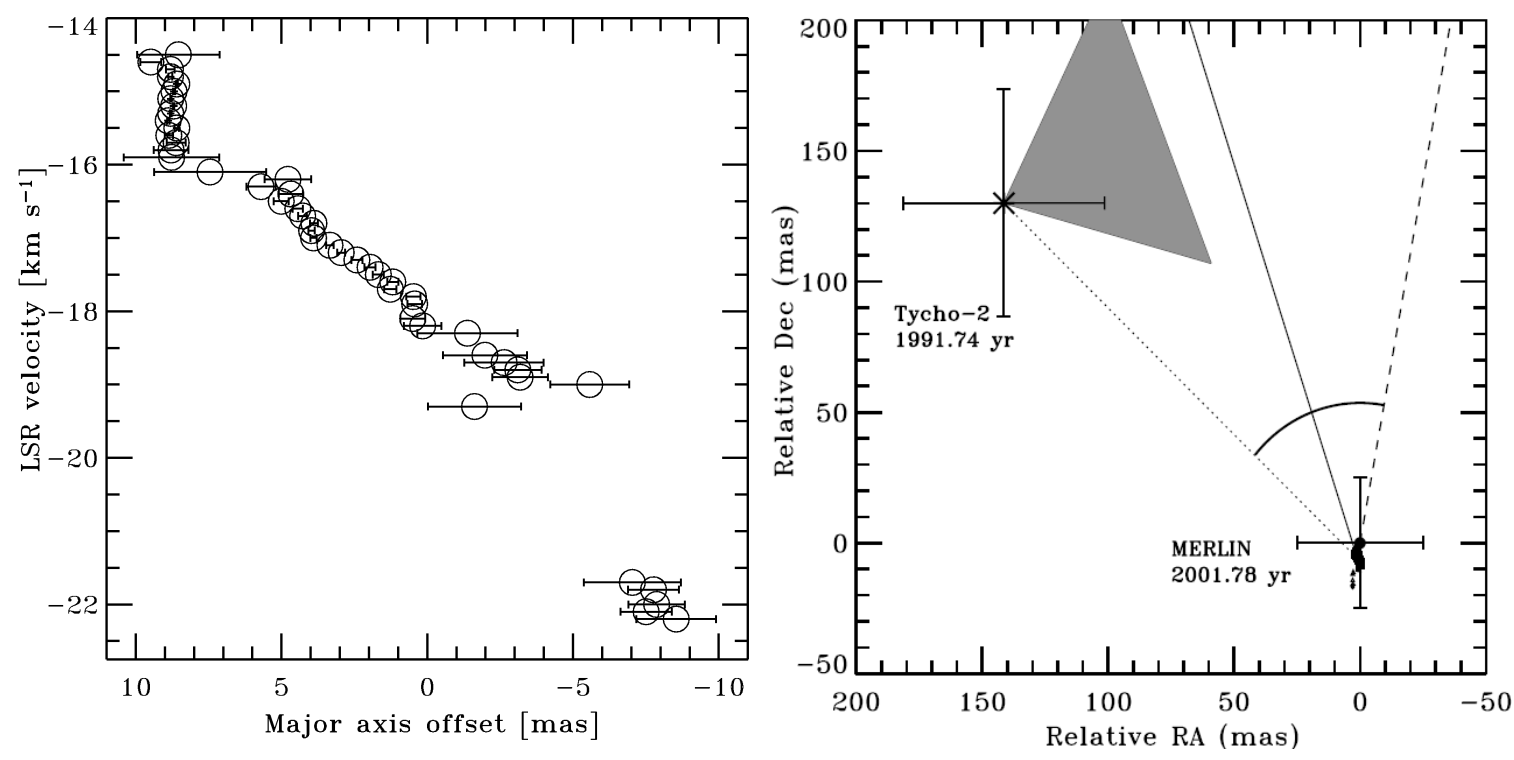

Figure 3: left The increment of velocity with position along the major axis of V778 Cyg. right Relative positions of the $C \star$ and water masers in 2001 . The former is constrained by the Tycho position and proper motion uncertainties (shaded region). The long dotted, solid and dashed lines show the limits of the orbital plane direction and the disc plane deduced from the brightest and from all masers, respectively.

\subsection{Masers associated with low- as well as high-mass star formation}

The first methanol and water masers to be detected in SFR were associated with high-mass YSOs and are often very bright (hundreds - millions Jy). It was assumed that only massive objects produced sufficiently strong outflows sufficiently rapidly to interact with the ISM in the "window of opportunity' for masing, after molecules have sublimated off grains but before the region becomes too ionised or is cleared by radiation pressure. In the last few years, more sensitive observations have revealed weaker masers around lower-mass YSOs.

NGC 1333 is a low-mass SFR; the nature of the YSOs has been well-studied using IR colours and continuum radio interferometry[5, 112]. Methanol masers at $44 \mathrm{GHz}$ (probably collisionally pumped) were found[78] located towards the outer lobes of outflows. Radiatively pumped 1.6-GHz $\mathrm{OH}$ and 6-GHz methanol masers were detected around IRAS20126+4104[37] in a disc orthogonal to the radio continuum jet, giving an enclosed mass of $\sim 5 \mathrm{M}_{\odot}$. Water masers have also been detected associated with low mass Class 0 protostars[54, 55] and VLBI monitoring suggests that they originate from outflows interacting with the ISM or discs.

Some of the first VLBI images of methanol masers were interpreted as Keplerian discs[95]; the fickle nature of maser amplification means that only part of a disc might be seen (making it hard to be sure if a low- or high-mass object is enclosed), or the structures might not trace a disc at all but arise from shocking outflows[35]. A disc presenting edge-on provides more effective maser amplification[105] but the disc hypothesis has been strengthened by a beautiful image of a face-on 6.7-GHz methanol ring (around a high-mass YSO)[11] now also seen at $12 \mathrm{GHz}[12]$, as well as water maser discs at small angles to the plane of the sky[56] (see Section 4.2). The recent evidence implies that water and possibly methanol masers could indeed be used to trace discs around low 
mass stars and might even reveal perturbations due to pre-planetary condensations. This suggestion first arose from analysis of velocity and intensity variations seen in long-term monitoring of singledish water masers[82, 84].

\section{Extended masers - discs and shocks}

\subsection{Extended $\mathrm{OH}$ and methanol in $\mathrm{W} 3(\mathrm{OH})$}

A chain of methanol and multiple transitions of $\mathrm{OH}$ masers has been detected in the massive star-forming region $\mathrm{W} 3(\mathrm{OH})$. Using 5 or 6 antennas between 4 and $7 \mathrm{GHz}$, MERLIN is sensitive to emission on scales from $<50$ mas to almost $1 \operatorname{arcsec}(<100-2000$ AU at the distance of W3(OH)). The contours in Fig. 4, left show very extended emission in the methanol[65] and 4.7$\mathrm{GHz} \mathrm{OH}[64]$ lines, tracing a filamentary structure extending at least $3100 \mathrm{AU}$, overlying an UCHII (ultra-compact HII region). The positions of ground-state $\mathrm{OH}$ masers[139, 140] follow the same line. The average velocity gradient is about $0.5 \mathrm{~km} \mathrm{~s}^{-1} \mathrm{AU}^{-1}$, seen in all species. It could arise from a massive disc around the $30 \mathrm{M}_{\odot}$ ZAMS (zero age main sequence) star which is thought to be the main exciting source in $\mathrm{W} 3(\mathrm{OH})$. The brightest methanol clump (peak at declination arcsec $\sim 25.2$ ) has a steep velocity gradient of $24 \mathrm{~km} \mathrm{~s}^{-1} \mathrm{AU}^{-1}$, suggesting a (separate) edge-on disc at a position angle of $55^{\circ}$. This gradient is also seen in the 12.2- $\mathrm{GHz} \mathrm{OH}$ masers[97], where, however, it was interpreted as a rotating biconical outflow.

Observations on the 100-mas scale show that many $\mathrm{OH}$ transitions and methanol appear to be spatially associated, but VLBI and 4-7-GHz MERLIN data demonstrate that the coincidences break down on scales of 15 mas or less, whilst the very extended diffuse methanol and 4.7-GHz $\mathrm{OH}$ alternate every few hundred mas. Many $6-\mathrm{GHz} \mathrm{OH}$ masers are co-located with $1665-\mathrm{MHz}$ $\mathrm{OH}$, but mostly have small but significant separations from methanol masers (Fig. 4, right, see key) and the 4.7-MHz OH masers avoid other transitions on the smallest scales (Fig. 4; [92]). The ground-state $\mathrm{OH}$ transitions are expected to be quenched at lower combinations of $n T$ [124] - but the observed velocity dispersions are, in fact, similar in regions supporting masers from different energy levels. The 4-7-GHz OH and methanol transitions occur together due to radiative pumping under very similar excitation conditions [26, 27], a model supported in $\mathrm{W} 3(\mathrm{OH})$ by the occurrence of the predicted combinations[60] of emission and absorption in a plethora of $\mathrm{OH}$ lines[62, $13-$ $15,139,140,64,49]$. Methanol is formed on grains in the ISM and sublimates off, along with water (the parent molecule for $\mathrm{OH}$ ), during star formation. The methanol abundance may reach $n$ required for masing before $\mathrm{OH}$ is formed from water[26] but on the other hand a deeper velocitycoherent column density of methanol is required[124]. The maser strengths are very sensitive to abundances so chemical differences may explain the small-scale anti-correlations.

\subsection{Water masers marking a CO cloud collision?}

The star-forming region S128 is $\sim 17 \mathrm{kpc}$ from the Galactic centre, where spiral density waves are ineffective and there are no known SNe to trigger star formation. The likeliest explanation for the observed HII regions[70], water masers[67] and YSO[16] is the collision of two giant CO clouds[67], shown by the dashed line in Fig. 5 (left). The water masers in three regions up to $\sim 1.3$ pc apart, were imaged using MERLIN in 1998 and 1999[111], following long-term spectral 

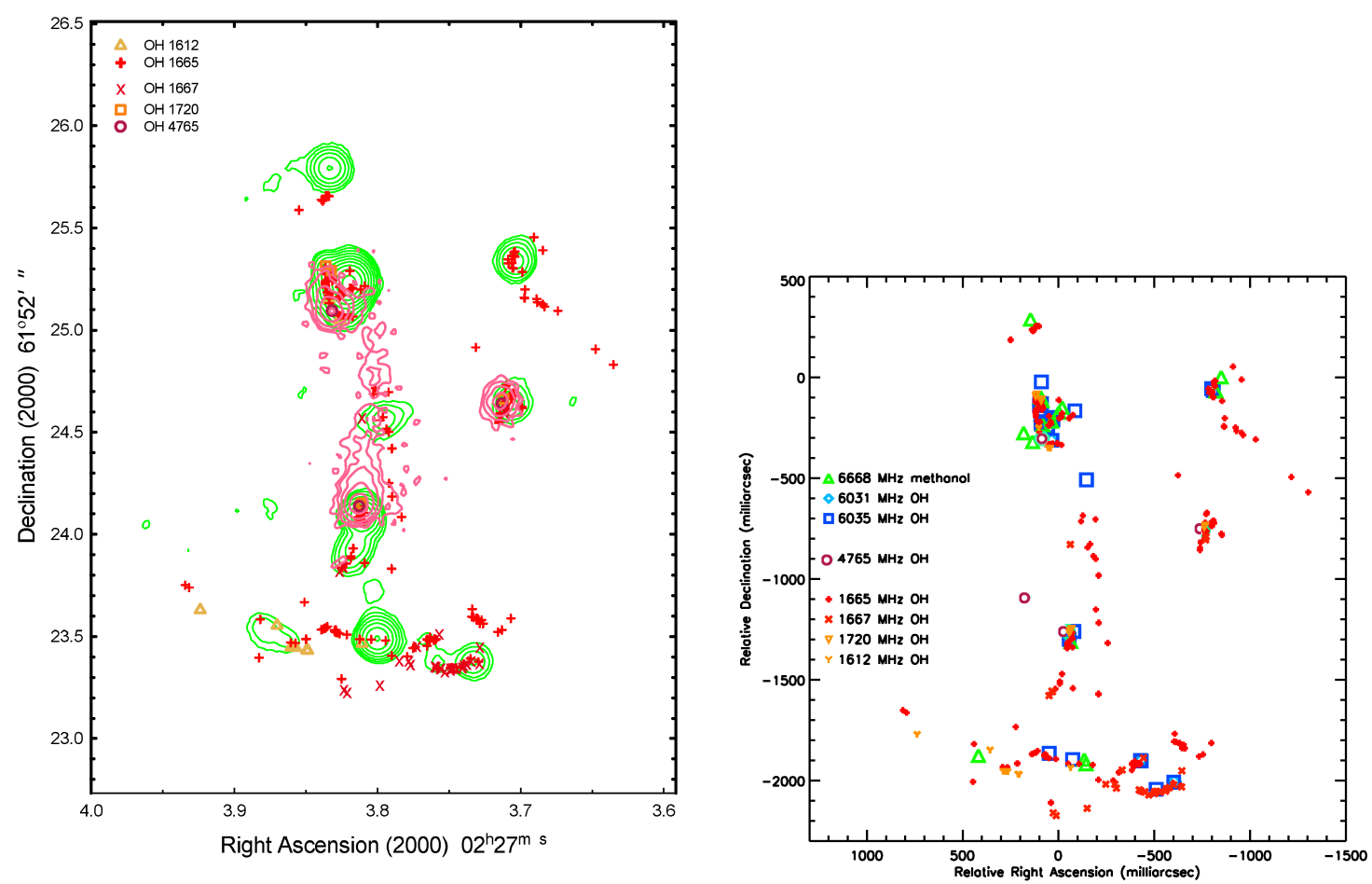

Figure 4: left Methanol (green) and 4.7-GHz OH (pink) contours overlying the ground-state $\mathrm{OH}$ maser positions (red and orange symbols)[65, 139, 140] right Positions of 4-6-GHz masers[49] and 1.7-GHz masers[139, 140].

monitoring[83]. Some lie close to the UCHII regions and/or have the distinctive morphology of bow shocks from YSO jets. A 900-AU filament is also seen (Fig. 5 right); it has a shorter parallel companion at about 350-AU separation and is elongated in a direction similar to the $\mathrm{CO}$ front but has no other obvious source of excitation. It exhibits a $15-\mathrm{km} \mathrm{s}^{-1}$ radial velocity dispersion with no systematic gradient but some series of components have a similar appearance at both epochs, displaced as if by a proper motion of $100 \mathrm{~km} \mathrm{~s}^{-1}$. It is possible that these masers trace the CO cloud collision front and the apparent proper motions are in fact the pattern speed of shocks (viewed approximately perpendicular to the shock direction[40]). The maser velocities in this region extend from similar to the $\mathrm{CO}$ velocities to more blue-shifted, suggesting that the more blue-shifted, Westerly cloud is pushing forwards as well as sideways into the Easterly cloud (which is presumably slightly closer to us). The fractal scales of this maser region, especially in velocity, are inconsistent with Kolmogorov turbulence[111], also suggesting a supersonic shock.

\subsection{Shocks in circumstellar envelopes and PPNe}

The masing regions in circumstellar envelopes are generally confined within a radius of a thousand $\mathrm{AU}$ at most, concentrated in small clumps. MERLIN is able to resolve water maser clouds out to a few kpc, whilst still detecting all the single-dish flux[109, 8, 100]). The average appearance and beaming properties of these clouds are usually similar to the spherical maser model of Fig 1 right (see Section 5.1) - but with some exceptions. U Ori, like many other AGB stars, 

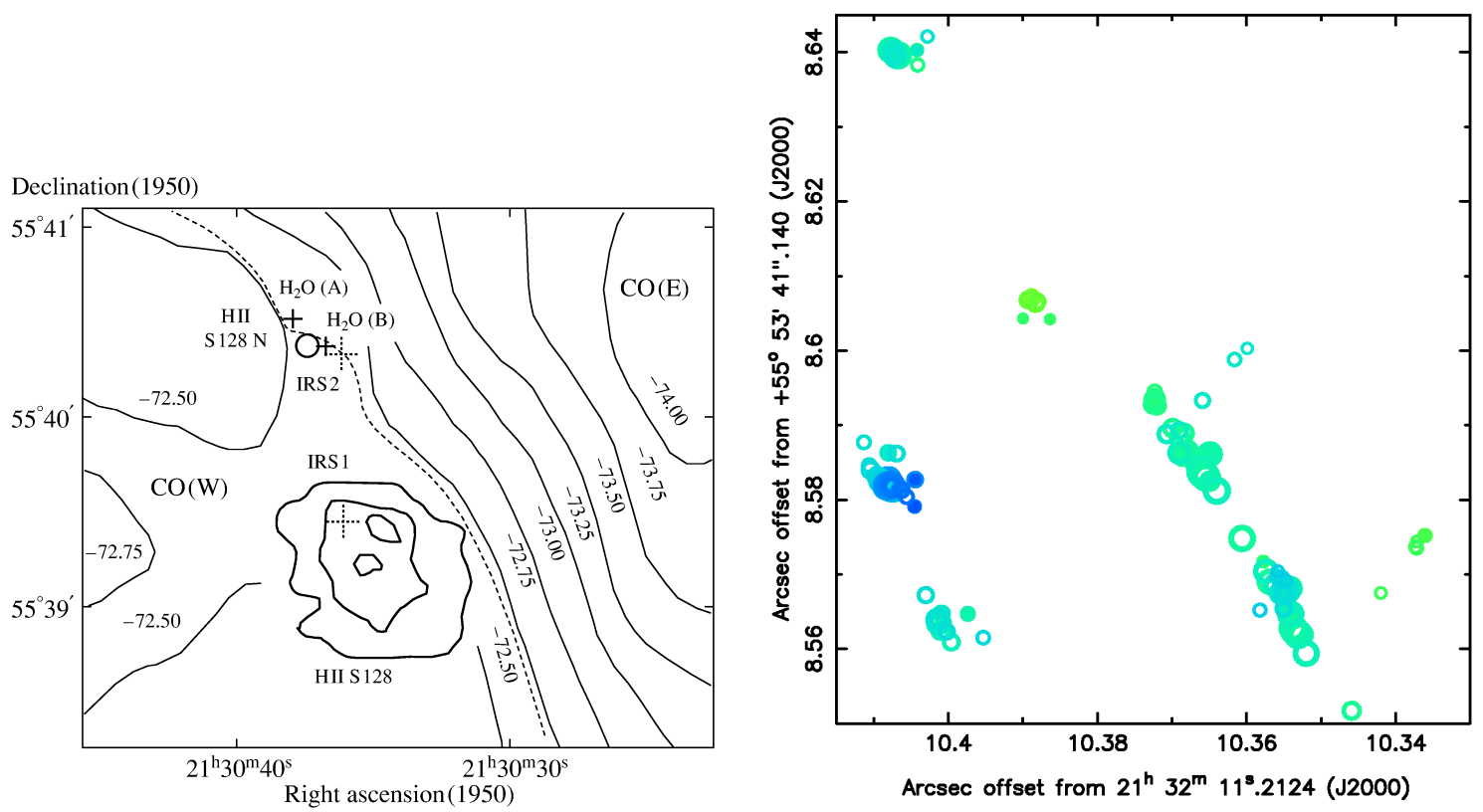

Figure 5: left Overview of S128[83]. The long dashed line shows the CO cloud collision front[67] and the circle marks the UCHII region. The solid + signs mark the two groups of water masers which have been visible for at least $20 \mathrm{yr}$; MERLIN also detected a third clump about half an arcmin to the $\mathrm{S}$ which has appeared more recently. right Part of water maser region B; the filled and hollow symbols show components detected in 1998 and 1999 respectively. The colour range shows velocities from $-82.5<V_{\mathrm{LSR}}<-72.5 \mathrm{~km}$ $\mathrm{s}^{-1}$. It is projected against the region where the CO clouds appear to interact.

exhibits drastic changes from year to year in its water maser shell morphology, including in the location of the most red- and blue-shifted features (Fig. 6 left) (illustrating the danger of deducing a bipolar CSE morphology from a single observation). Four epochs of MERLIN observations from 1994-2001 show that the water masers always fall within a hollow shell bounded by radii of $\sim 8$ and $\sim 35 \mathrm{AU}$, wherein the brightest emission is always at a similar distance from the star, although at different position angles. The weighted mean velocity of the brightest masers has drifted by less than $1 \mathrm{~km} \mathrm{~s}^{-1}$ in $20 \mathrm{yr}[113]$. Unusually, the apparent size of individual bright maser components is not inversely proportional to the brightness temperature as expected for beaming from approximately spherical clouds, but increases, as shown in Fig. 6 (right). This suggests that the brightest emission originates from an extended shocked region[40] (see Fig. 1 left).

VLBA measurements of circular polarization[131] reveal a very strong $(\sim 0.35 \mathrm{~T})$, complex magnetic field in the water maser region, possibly enhanced by shock compression (see Section 4.2). U Ori has also experienced an unusual 1612-MHz maser flare[57] - possibly also a symptom of shock dissociation of $\mathrm{H}_{2} \mathrm{O}$. The shocks could originate from the deep, regular stellar pulsations of U Ori[1].

As AGB stars make the transition towards Proto-Planetary Nebulae (PPNe), the CSE becomes more axisymmetric and the appearance of $\mathrm{OH}$ masers in many PPNe can be modelled as a biconical outflow emerging from an older, more spherical shell[143]. This is seen in MERLIN images of $\mathrm{OH}$ 17.7-2.0 [7] and Nançay telescope monitoring recently detected a flare in one part of the $\mathrm{OH}$ 

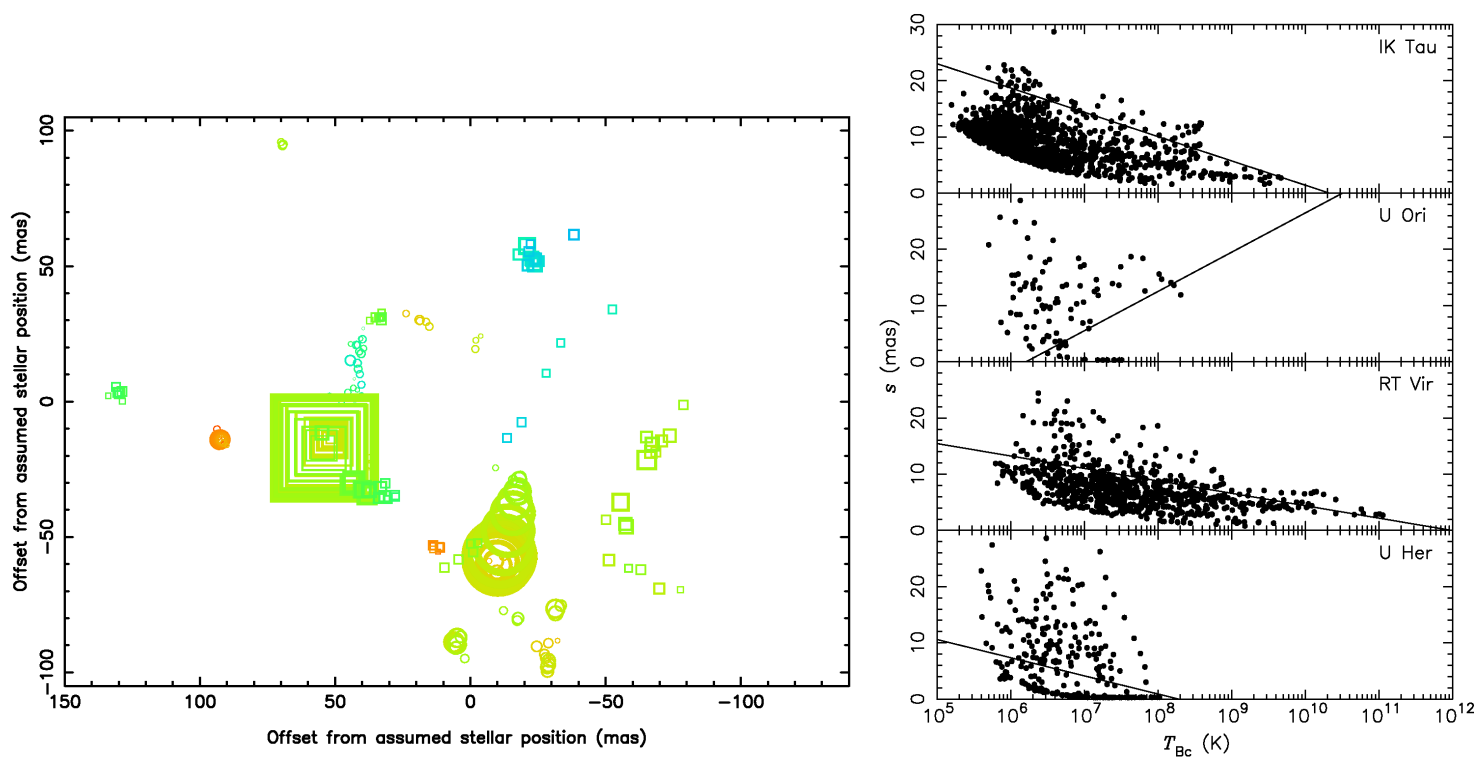

Figure 6: left Water maser components in the CSE of U Ori observed in 1994 (circles) and 1999 (squares); the size is proportional to the flux density and the shade from red to blue indicates the velocity range -36 $--43 \mathrm{~km} \mathrm{~s}^{-1}$. right Maser (beamed) component size as a function of flux density for four AGB stars. The cut-off at smaller sizes for fainter spots is a selection effect but only U Ori shows systematically larger components at higher brightness temperatures.

spectrum[125]; EVN images show that this was also spatially localised. This could arise from the impact of the shock front on an individual $\mathrm{OH}$ clump (likely to enhance the magnetic field, see Section 4.2) or from two clumps overlapping along the line of sight. The size-brightness relationship of individual maser components could also distinguish between these scenarios.

\section{Masers as probes of magnetic fields}

Maser lines from non-paramagnetic species such as methanol and water only undergo slight Zeeman splitting (much less than the thermal line width) even in the presence of a relatively strong magnetic field (e.g. $1 \mu \mathrm{T}$ ). There has been a recent upsurge in detections of very small fractional polarization (circular and/or linear) thanks to high resolution (to avoid beam-averaging depolarization) and high sensitivity (for a good signal to noise ratio in small beams and narrow spectral channels), including the first methanol polarization images[133, 66] and the first maps of the magnetic field around evolved stars using water masers[128, 129, 131]. The EVN and MERLIN both now have good baseline coverage at $6 \mathrm{GHz}$, covering paramagnetic excited $\mathrm{OH}$ transitions as well as methanol. The inclusion of the Lovell and Effelsberg alongside the 32- and 25-m telescopes in the EVN, and the use of eVLBI network tests, means that it is now a sensitive and reliable polarimeter. We are currently close to a period of minimum Solar activity; if polarization observations are to continue for the next decade we must improve techniques for correcting for ionospheric variability, whether by using GPS, more sophisticated self-calibration or any other means (as is being discussed by RadioNet, NRAO and others). 


\subsection{Controversy}

Interpretation of Zeeman splitting and of the observed linear polarization angles remains controversial[61]. The L-R velocity difference of $\mathrm{OH}$ is often greater than the thermal line width in star-forming regions so the strength and direction of the line-of-sight magnetic field component can be determined unambiguously. In circumstellar envelopes, the velocity difference is much smaller and some models (e.g.[136]) predict a much smaller field strength than that obtained by extrapolating from the large-splitting case. Observations suggest that this is unlikely to be a severe effect as even the highest magnetic field estimate using $\mathrm{OH}$ under such conditions is smaller than the strength inferred from other species, see Sections 4.2 and 4.3.

Deducing the magnetic field properties from linear polarization observations is even more fraught. This relies on a number of assumptions, such as:

1. Whether $\sigma$ or $\pi$ components are detected for $\mathrm{OH}$. The former are displaced in velocity from the unpolarized emitting-frame rest frequency and show circular or elliptical (i.e. mixed linear+circular) polarization; the latter appears at the rest frequency and is $100 \%$ linearly polarized. All three components have only been detected at one location in W75, by two independent observations using different instruments[76, 51]. In other cases, a pair of series of components are detected with spectral offsets consistent with the displacement expected for $\sigma$ components in a feasible magnetic field. Very often, however, components are detected in one hand of circular polarization only, or with very unequal intensities between left and right but it seems reasonable to interpret these as $\sigma$ components in an environment where one component is suppressed by the interaction of velocity and magnetic field gradients[24] in a clumpy medium.

2. Whether Faraday rotation is significant. The polarization angle of radio emission is rotated by propagation through an ionised medium. A uniform electron screen between the observer and the source produces a frequency-dependent rotation but inhomogeneities on the scale of the observing beam will smear and reduce the detectable linear polarization. This also occurs if the maser is itself propagating in a medium with a significant ionisation fraction, supposedly liable to convert $\sigma$ and $\pi$ linearly polarized components to circular $\sigma$ components [59]. Some $\mathrm{OH}$ observations do show an almost incomprehensibly complicated picture, which supports this model[51], but makes it difficult to deduce the intrinsic magnetic field direction in the plane of the sky. In other cases, however, a coherent picture does emerge, (see Section 4.4.

3. The magnetic field orientation to the line of sight. The linear polarization angle varies with the angle $\theta$ between the magnetic field and the line of sight with a discontinuity at the critical angle $\theta \sim 55^{\circ}[41]$. This was initially regarded with suspicion as unphysical but in several sources, either such a discontinuity has been observed (e.g. see Section 5.1) or there is separate evidence supporting the interpretation of linear maser polarization vectors in one or other magnetic field regime (e.g. see Sections 4.3 and 4.4). The exact dependence with respect to the maser saturation state, the velocity gradient and the magnetic field strength as well as direction, remains controversial[136, 132]. 
In short, the range of $\mathrm{OH}$ and other transitions now accessible, and in some cases continuum measurements, has allowed self-consistent pictures to emerge for some objects but there is less agreement as to the underlying mechanisms.

\subsection{Density-dependent field strength in SFR}

The strength of a frozen-in magnetic field depends on the number density as $\sim n^{0.5}$ [98]. This helps to explain a number of observations of SFR, for example the increase of the magnetic field strength with age for $\mathrm{OH}$ masers in YSO discs in NGC7538[77] as the field lines are wound up[127] - but the decay of field strength with time in Cepheus A where an $\mathrm{OH}$ maser is located in gas expanding around a very young protostar[10]. Clearly, good resolution is required to distinguish such situations.

Shock compression also reconciles the difference in field strength measured from $\mathrm{OH}$ and $\mathrm{H}_{2} \mathrm{O}$ masers in Cepheus A. This contains a ring-like water maser structure (region R4[126]) with a radius $\sim 50 \mathrm{AU}$. Multi-epoch observations suggest that this is consistent with an expanding but decelerating disc $[56,135]$. The polarization vectors are mostly tangential to the disc except for a few with a $90^{\circ}$ discrepancy, implying that the magnetic field is at close to the critical angle of $55^{\circ}$ to the line of sight. The magnetic field strength inferred from water maser circular polarization[135] is several tens of $\mu \mathrm{T}$ with several reversals in field direction on sub-AU scales. This suggests that the magnetic field is frozen in to clumps and the line-of-sight component at least has become tangled in shocks. The proper motion measurements suggest a shock velocity of $10 \mathrm{~km} \mathrm{~s}^{-1}$ which could produce the observed field strength by compression of a field enhanced by a stellar dynamo. The magnetic field can be measured for both $\mathrm{OH}[10]$ and water masers in a nearby region where the outflow from HW2 interacts with the ISM. The number densities required to support $\mathrm{OH}$ masers are about $1 / 50$ of the density for water masers and shock compression of the $\mathrm{OH}$ gas would give the observed enhancement of the magnetic field from 1-1.5 $\mu \mathrm{T}$ to approaching $7 \mu \mathrm{T}$, as observed in the $\mathrm{OH}$ and water maser regions, respectively[135].

Shock compression is a likely explanation for the recent $\mathrm{OH}$ flare in $\mathrm{W} 75(\mathrm{~N})$ and also for the amplification of the the magnetic field to $40 \mathrm{mG}[116,2]$ but the effects of a magnetized YSO and/or protoplanetary disc could also be factors in this case.

\subsection{Mysterious magnetic fields around evolved stars}

Resolved polarimetry for all of $\mathrm{SiO}, \mathrm{H}_{2} \mathrm{O}$ and $\mathrm{OH}$ is now available for a significant number of CSEs and is converging on a picture where the magnetic field $B$ falls off from roughly $\mathrm{mT}$ close to the stellar surface, to tens of nT at a distance $r$ of $1000 \mathrm{AU}$ or further from the star[131, 68]. This can be expressed as $B \propto r^{-\alpha}$ but there is no consensus as to the value of $\alpha$ except that it probably lies between 1 and 3 . The field configuration has been modelled convincingly in a few individual objects e.g. as a dipole in VX Sgr[123, 131] and some in post-AGB stars and PPNe e.g. OH17.7-2.0[7] but as a toroidal field in IRAS 20406+2953[9]. Deviations from a smooth power law (whatever the exponent) may be an inevitable consequence of the clumpiness of the wind; even at the same distance from the star different density clouds may represent different degrees of magnetic field enhancement (Section 4.2). This is consistent with other data on, for example, $\mathrm{S}$ Per, see Section 5.1. 
Although the masing regions of CSEs are usually modelled as neutral gas and dust, there is evidence for a significant ionised fraction. A shift in the linear polarization angle between red- and blue-shifted $\mathrm{OH}$ from W Hya was interpreted as due to a fractional free electron density $\sim 2 \times 10^{6}$ $\mathrm{m}^{-3}$ [120]. This can also cause depolarization and in fact linear polarization is only detected from blue-shifted emission in W Hya[120], R Crt[121] and S Per (Section 5.1).

The origin of the stellar-centred magnetic field is still a mystery. The vast expansion of the stellar atmosphere when stars ascend the AGB is thought to reduce the surface rotation to a negligible value. This may conceal a rotating white-dwarf-like core but there is no convincing model of how a structured magnetic field, but not angular momentum, could be transported into the wind. The rôle of such a core could be tested by seeing whether the magnetic field interpolated to a given distance (e.g. a few AU) appears to increase as the star evolves towards the PN stage and the core is increasingly exposed. There is a more fundamental problem, though: RSG possess similar magnetic fields but cannot have a degenerate core until much later evolution into a supernova!

Stellar convection might give rise to locally magnetised clumps[36, 63], susceptible to ordering by a weak dipole [117] but it is claimed that the resulting field would be insufficient to shape outflows from late-type stars. Nonetheless, magnetic fields are the only observable phenomenon that do provide sufficient energy density to contribute to shaping the outflow $[19,120,121,7,131]$ or even collimate a PPNe jet[134]. In some cases, e.g. VX Sgr, multiple separate observations support the alignment of the kinematic[100] and magnetic axes. A similar magnetic field configuration was deduced from $\mathrm{OH} 1612-\mathrm{MHz}[123]$ and $\mathrm{H}_{2} \mathrm{O}$ [131]. In contrast, there is no evidence for a dynamically significant companion in the great majority of evolved stellar maser sources; the few exceptions display distinctive maser properties, e.g. V778 Cyg (Section 2.2), OH 231.8+4.2[91], $o$ Cet and R Aqr[25], or are the most extreme bipolar PNe. Alternative mechanisms involve the presence of (so far undetectable) planetary and/or debris discs but proper motion studies place upper limits $<1 \mathrm{~km} \mathrm{~s}^{-1}$ for any rotation in most AGB and CSE winds e.g.[21, 110, 100].

The physics of how a magnetic field channels the wind are also non-obvious. The DavisGreenstein effect[30], whereby dust grains are deflected along field lines, does not apply in CSE where grains are the main absorbers of stellar radiation and are thus hotter than the gas. It is possible that magnetic forces act preferentially on lower density, optically thinner and therefore more ionised gas - such as the bicones of VX Sgr and many PPNe[143]. This is hard to reconcile with the higher fields measured in water maser clumps[129, 131] (Section 5.1).

\subsection{Supernova remnants}

$1720-\mathrm{MHz} \mathrm{OH}$ emission from a series of SNR has been imaged using single dishes and the VLA, MERLIN and the VLBA. This wide range of instruments now available (including full polarization facilities) provides a complete picture of the masing gas and the magnetic field which it experiences from pc to AU scales. The 1720-MHz maser transition is a unique tracer of cool, fairly diffuse gas $\left(50<T<125 \mathrm{~K}, n \sim 10^{11} \mathrm{~m}^{-3}\right)$, collisionally excited when a SN shock front interacts non-dissociatively with a dense molecular region of the ISM[53, 88]. OH absorption was detected from IC 443 over a much larger region than the maser[71], probably due to unfavourable orientations of the exciting shocks[69]. In W28, the masers trace an arc, (also seen in CO[4]), which is aligned with the expanding shock front[73], see Fig. 7. The maser polarization angle is consistent with that of the radio synchrotron emission from the shock[18]. In W44, however, the maser po- 


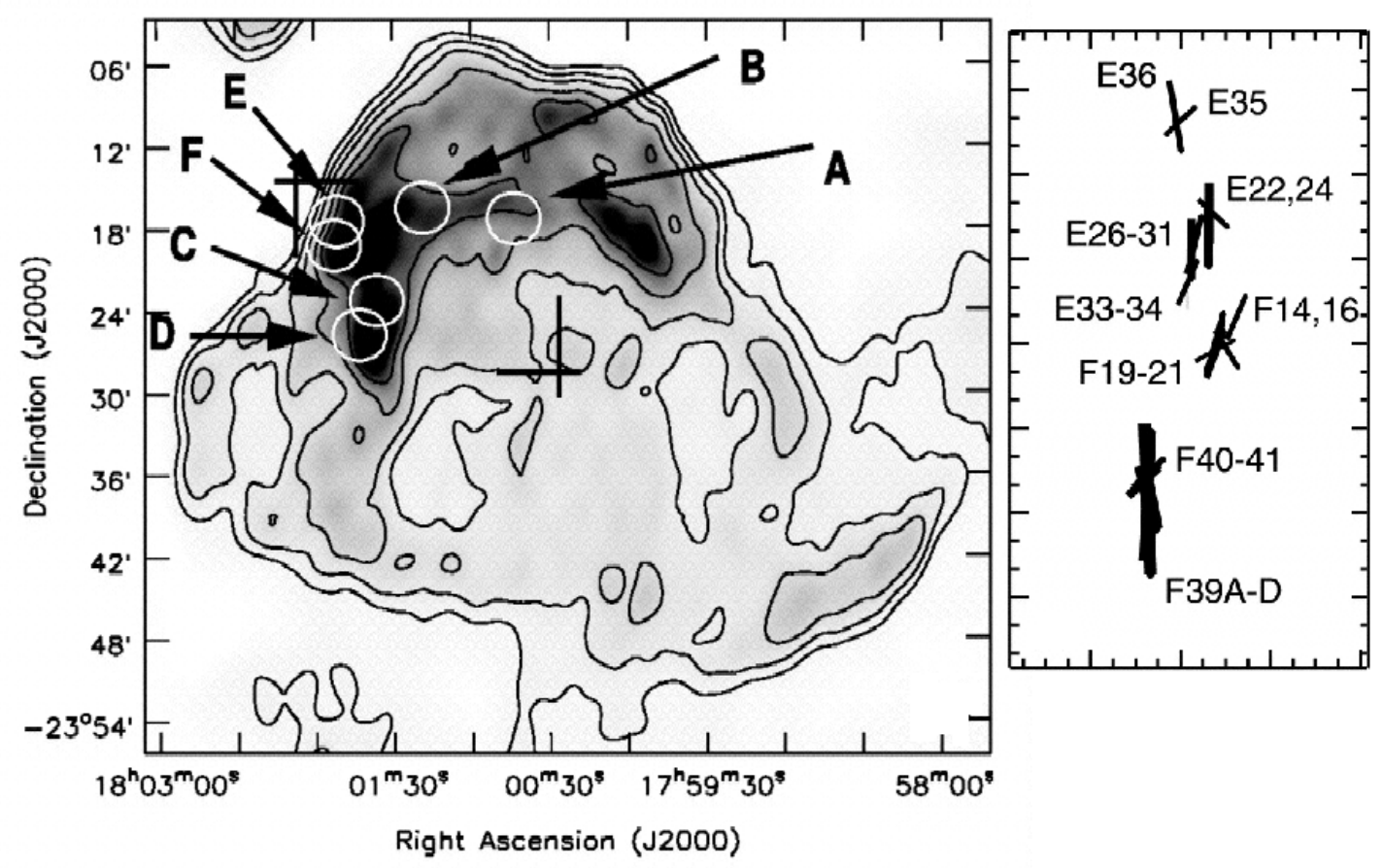

Figure 7: The left-hand image shows radio-continuum from the SNR W28 with the OH 1720-MHz maser positions marked. An enlargement of regions $\mathrm{E}$ and $\mathrm{F}$ is shown on the left, demonstrating how the maser magnetic field position angles trace the shock.

larization angle[74] is perpendicular to the synchrotron polarization vectors[94], measured at more than one frequency to allow compensation for Faraday rotation. A possible explanation is that in in W28 the magnetic field angle to the line of sight exceeds the critical angle of $55^{\circ}[42,136]$ (and see Section 4.1), whilst in W44, the magnetic field orientation is $<55^{\circ}$ to the line of sight and so the maser polarization vectors are perpendicular to and aligned with the magnetic field component in the plane of the sky, respectively[74].

\section{Mass loss from AGB and RSG stars?}

The most detailed studies are possible where multiple maser species can be resolved from the same CSE. These reveal a far more complex picture than the traditional 'onion' model, with its orderly sequence of spherical $\mathrm{SiO}, \mathrm{H}_{2} \mathrm{O}$ and $\mathrm{OH}$ maser shells. $\mathrm{SiO}$ maser monitoring shows both expansion and infall within a few stellar radii $(R \star)$ [21], probably driven by stellar pulsations[17]. Dust appears at $4 R \star$ and beyond[28]; IR interferometry[29, 96] shows that it can be clumpy, episodic and/or asymmetric, in some cases forming multiple shells possibly on the timescale of 'superperiods'[115]. Multiple maser shells are also seen in e.g. NML Cyg[48].

Water masers show more systematic acceleration radially outwards from the star (between $\sim 5--\sim 25 R \star[142,108,107,110,109,8,100])$. OH mainline masers are found at similar distances from the star, with their inner radius typically in the centre of the water shell. This requires revision of the original model wherein $\mathrm{OH}$ is confined to an outer layer where external 


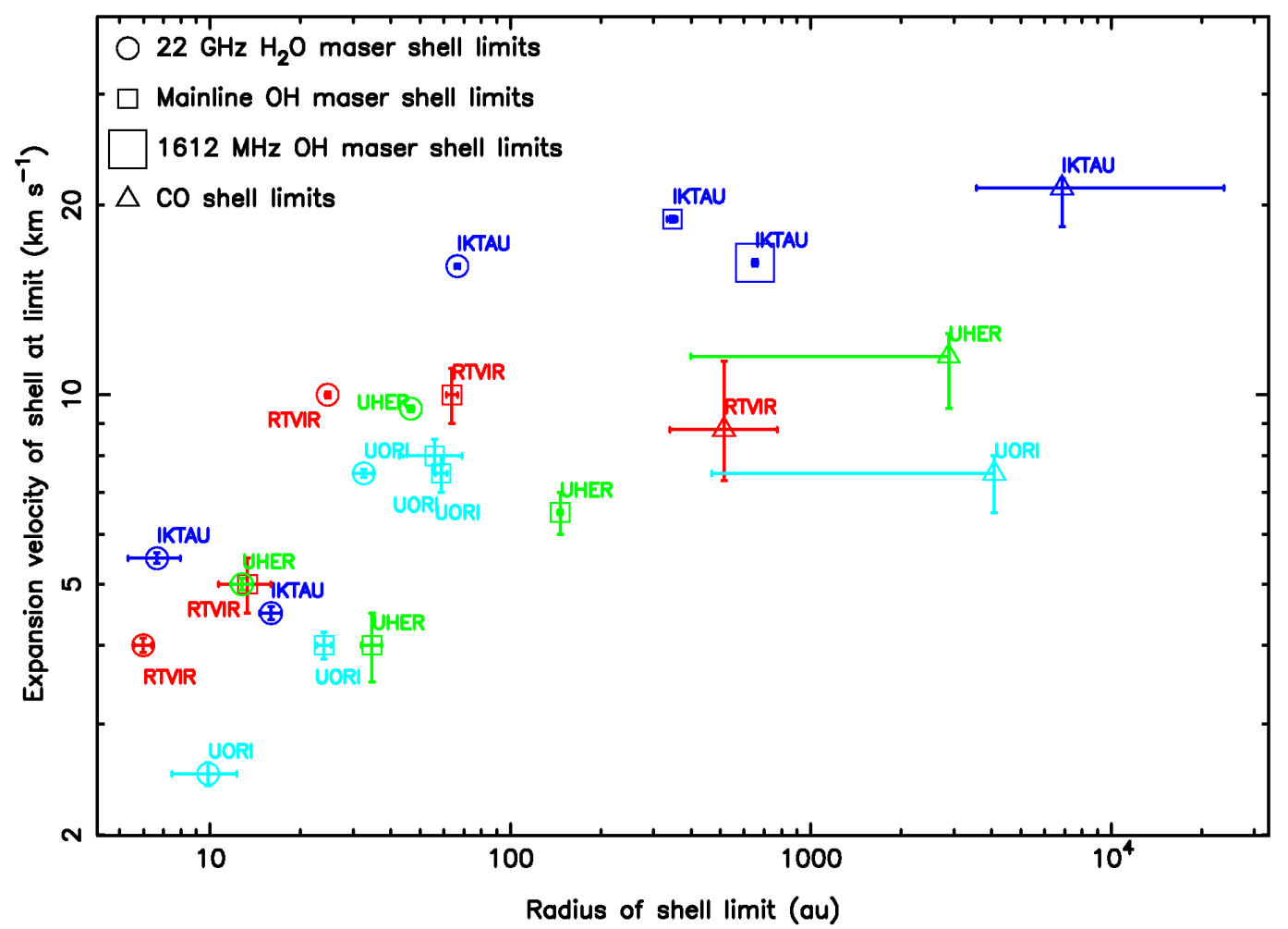

Figure 8: Expansion velocity as a function of distance from the star for four AGB stars.

UV penetrates the outer layers of the CSE to dissociate $\mathrm{H}_{2} \mathrm{O}$. It also provides a severe constraint on pumping schemes, especially as excited $\mathrm{OH}$ has not been detected down to limits of a few percent or less of the ground-state mainline peaks. The problem is mitigated by the structure and kinematics of the region, however, since the collisionally pumped water masers are concentrated in clumps which are typically $50 \times$ denser $\left(n \sim 10^{15}-10^{16} \mathrm{~m}^{-3}\right)$ than the average wind density measured from $\mathrm{CO}$ and other thermal emission. The $\mathrm{OH}$ mainline masers appear to come from the lower-density interclump gas and tend to have a slower expansion velocity at a given radius[8] (Fig. 8). As both heating and acceleration are driven by radiation pressure on dust, transferred to the gas in collisions, the $\mathrm{OH}$ medium could be at about $500 \mathrm{~K}$ (half the water clump temperature), just cool enough for the radiative pump mechanism (Gray, priv.comm.).

OH 1612-MHz masers are usually found at greater distances, consistent with the cooler conditions and larger velocity-coherent column densities required for amplification. They also frequently deviate from the classic spherical model for thick-shelled OH/IR stars, for example elongated outflows seen in IK Tau[80], NML Cyg[48] and S Per (see below). In addition, the velocities of rare $1612-\mathrm{MHz}$ flare emission [57, 46] indicate that in at least some cases this may emanate from the inner CSE, possibly due to shocks (Section 4.2). 

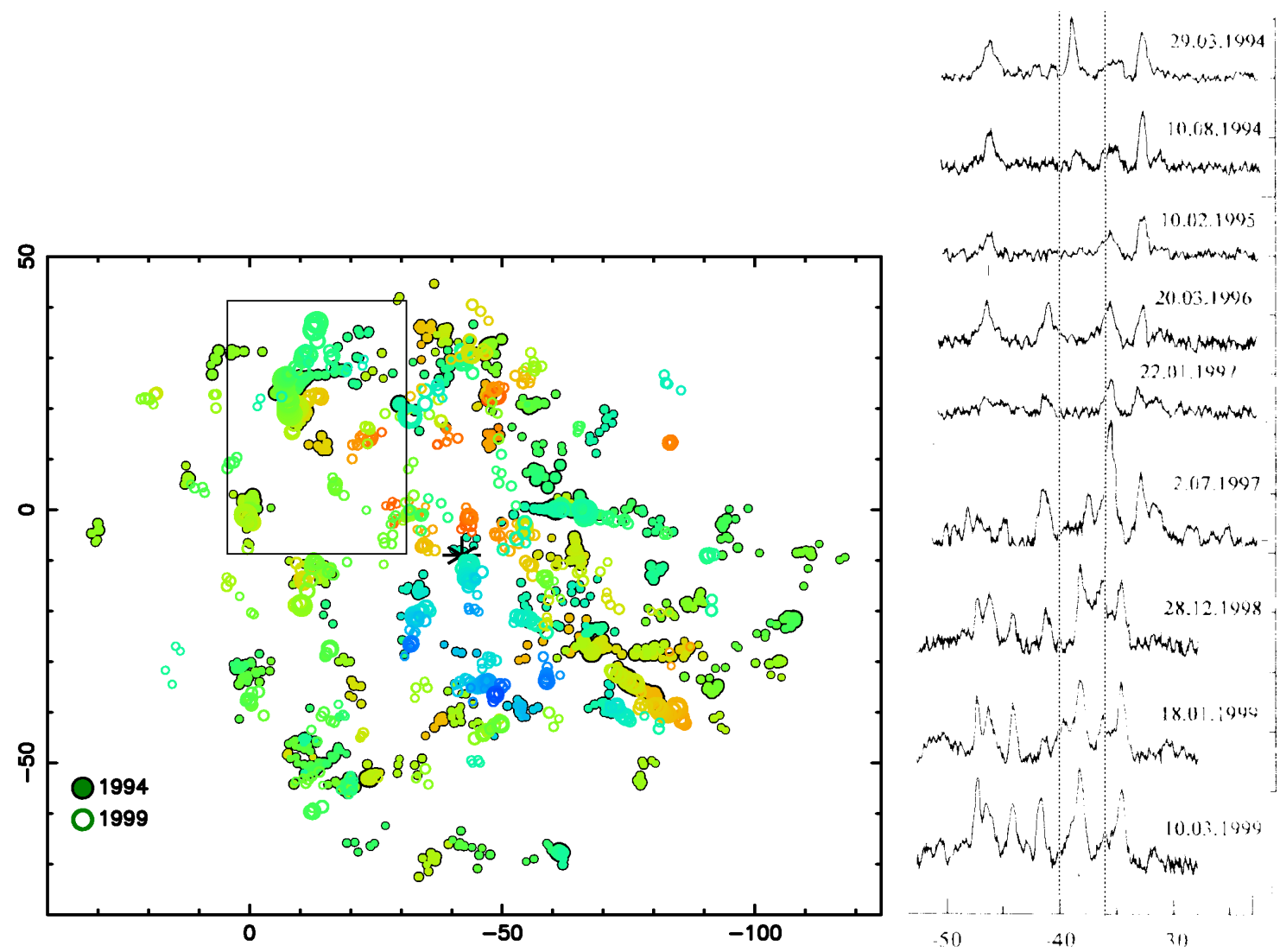

Figure 9: Monitoring water masers around S Per. left The boxed region shows bright maser components peaking between $-37--40 \mathrm{~km} \mathrm{~s}^{-1}$. The pattern seen in 1994 (March) can be identified, partially twisted northwards, in 1999 (January). The peak velocity lies between the dotted lines in the spectra right, showing that the feature disappeared soon after the MERLIN observations in 1994 and reappeared by 1998.

\subsection{A fuller picture of clumpy mass loss - S Per}

The high-declination red supergiant S Per is ideally placed for study by MERLIN and VLBI. $\mathrm{SiO}$ monitoring has been carried out using the VLBA[104]. MERLIN has been used to image more or less all the single-dish emission from $\mathrm{H}_{2} \mathrm{O}$ and $\mathrm{OH}[109]$ and has been used to map the $\mathrm{OH}$ masers in full polarization. Only the brighter masers are detected at VLBI resolution but this has been used to measure the water maser polarization[128, 129] and image $\mathrm{OH}$ masers[89] at a resolution matching MERLIN at $22 \mathrm{GHz}$. This has been complemented by single dish monitoring such as in the water line[85]. Results below are taken from these references and from unpublished MERLIN data (unless otherwise stated).

Figure 9 left shows the water maser distribution at 2 epochs 5 years apart. The masers form a hollow shell with inner and outer radii of about 5 and 15-20 $R \star$, respectively. The most redand blue-shifted masers are fainter and are seen in projection against the centre of the shell, whilst the brightest emission is close to the stellar velocity and is seen projected at larger angular separations, typical of an accelerating spherical thick shell. There is an NE-SW belt of slightly brighter masers, often described as an equatorial density enhancement. The masers are grouped in series of components, in many cases forming a similar spectral peak and spatial pattern (with a systematic 


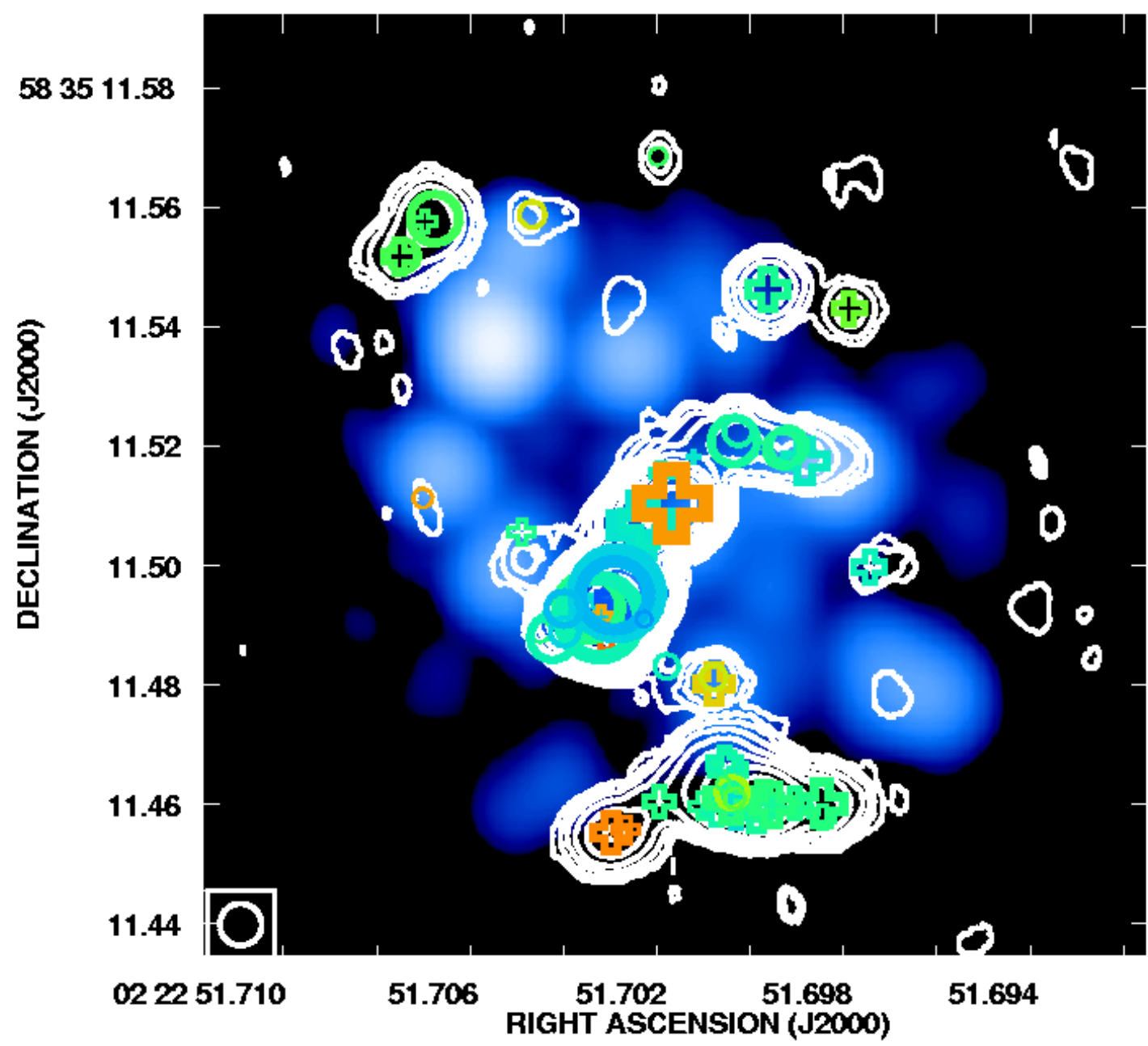

Figure 10: Water maser emission (MERLIN) from $\mathrm{S}$ Per is shown in blue overlaid with $\mathrm{OH}$ mainline contours (VLBI) and symbols indicating the velocity (shade), strength (size) and sign of OH Stokes V $(\circ \equiv-)$.

if not linear velocity gradient) at both epochs. The water masers form clouds of about 10-20 AU diameter (measured as shown in Fig. 1 right), similar to $R \star$; if the material has expanded monotonically with distance from the star, the cloud 'birth' radius was about $0.1 R \star$. Similar proportions are seen in other AGB and RSG shells $[8,100]$ despite the difference in $R \star$ (typically 1 and $10 \mathrm{AU}$, respectively).

In AGB and RSG CSEs, individual water maser clouds can be traced for, typically, less than a year and for a decade, respectively, a similar timescale to the crossing time of sub-sonic or mildly supersonic shocks for the average cloud radius in each case. This is less than $1 / 5$ of the kinematic crossing time for the entire water maser shell, presenting a conundrum: how do masers continue to be detected for so long? Single-dish monitoring (Fig. 9 right) shows that even where a distinctive maser clump was imaged in both 1994 and 1999, the corresponding spectral feature has faded and reappeared in the intervening years (and before and since). This implies that the clouds themselves 

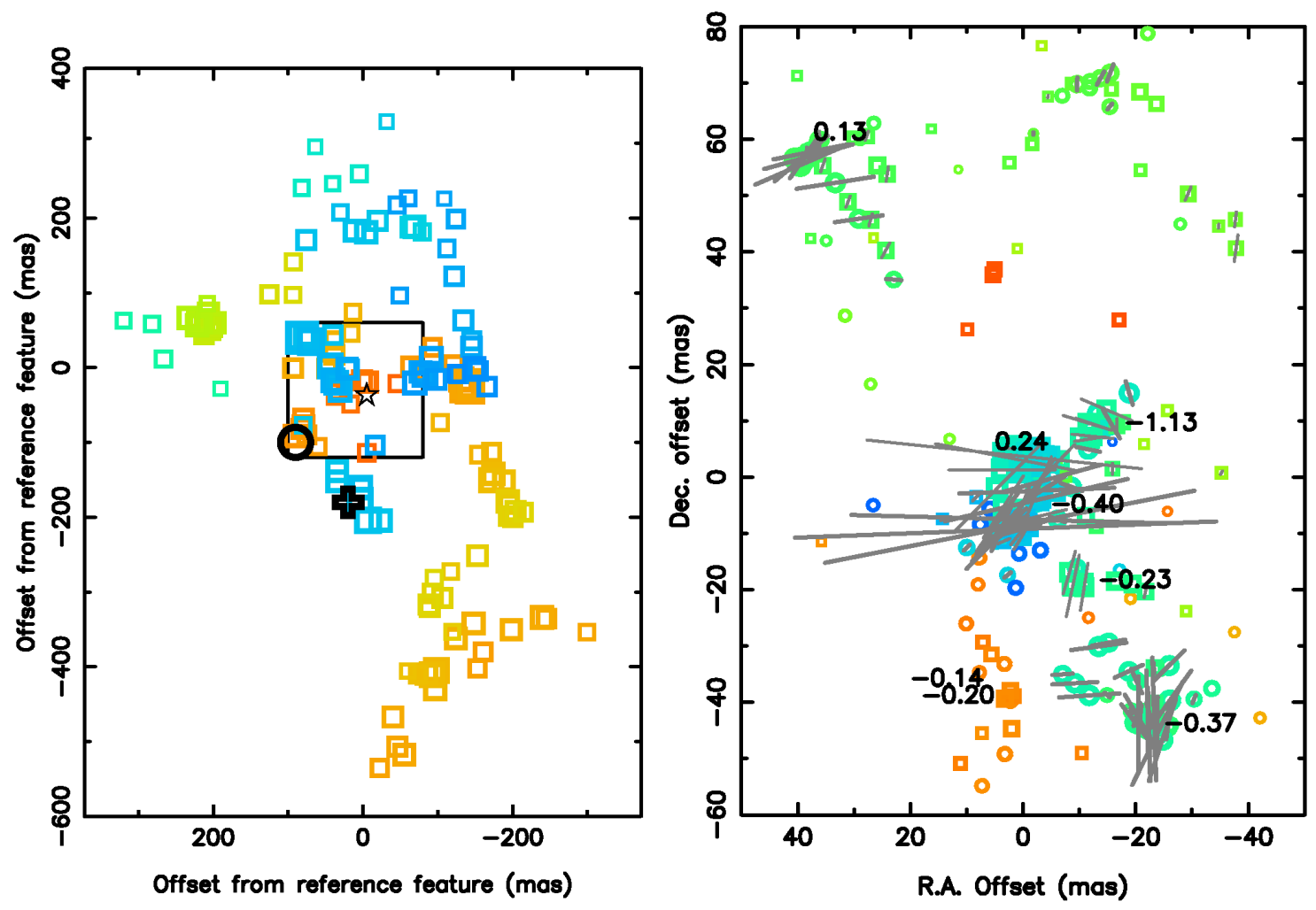

Figure 11: MERLIN OH maser components: left $\mathrm{OH}$ 1612-MHz maser emission from S Per; the only two polarized components detected are marked by the signs of Stokes V ( $0 \equiv-$ ). The OH mainline and water masers fit within the small box. right $\mathrm{OH}$ mainline masers at $1667 \mathrm{MHz}$ (circles; heavy vectors) and $1665 \mathrm{MHz}$ (squares, thin vectors) showing the relative velocity (shade), linear polarization direction and line-of-sight magnetic field strength in $\mu \mathrm{T}$.

retain their integrity for decades but whether or not we detect maser emission from any individual cloud at a given epoch is an accident of beaming orientation and/or local excitation conditions.

The $\mathrm{OH}$ mainline masers are found at similar distances from S Per but interleave the water masers, as shown in Fig. 10 and have a far less symmetric distribution, elongated NNE-SSW in an almost biconical structure. This is seen more clearly in Fig. 11 and the similarities between the $1612-\mathrm{MHz}$ and the mainline maser distributions despite the difference in scales suggest that the axisymmetry has persisted for over a century. Several Zeeman pairs were identified in either or both the MERLIN and VLBI data showing a magnetic field directed towards the observer in the $\mathrm{S}$ and away in the N. Linear polarization is only detected from blue-shifted masers, with vectors mostly perpendicular to the axis of symmetry except in the $\mathrm{S}$ where the angle between the magnetic field and the line of sight may pass through the critical angle (Section 4.1). The absence of redshifted linearly polarized emission suggests that there is an appreciable, turbulent population of free electrons, either in a screen in the plane of the sky containing the star, or inside the bicones suggested by the $\mathrm{OH}$ morphology[143].

The line-of-sight magnetic field strength measured from $\mathrm{H}_{2} \mathrm{O}$ masers[129] is $\sim 7-20 \mu \mathrm{T}$ (depending on location and model used). The brightest water masers with measurable polarization 
are probably slightly closer to the star than the average distance of the OH Zeeman pairs but even extrapolating to comparable radii the water masers seem to experience a magnetic field which is around ten times stronger than the field affecting the $\mathrm{OH}$ masers. The requirements for $\mathrm{H}_{2} \mathrm{O}$ and $\mathrm{OH}$ masing suggest that the former arise from clumps with a number density at least 50 times higher than that in the $\mathrm{OH}$ mainline regions or the wind average. The water and $\mathrm{OH}$ masers are predominantly tangentially and radially beamed, respectively, suggesting that the water molecules are more effectively accelerated by collisions with dust and therefore are in a denser environment. This implies that the magnetic field is frozen into the stellar wind at an early stage and enhanced by compression[98] (Section 4.2) when the water maser clumps become differentiated.

\section{Conclusions and future developments}

High-precision measurements of masers now provide detailed descriptions of the local environment and the properties of their host. Kinematics are revealed on scales from sub-AU proper motions to improved distances based on Galactic rotation. On the large scale, improved singledish efficiency has uncovered masers in surprising places, from globular clusters to dark corners of star-forming regions; interferometric follow-up will be vital, for example to align new MMB (Methanol Multi-Beam, [23]) and GLIMPSE (Galactic Legacy Infrared Mid-Plane Survey Extraordinaire, [58]) sources.

In SFR, masers are associated with both outflows and discs but the common factor seems to be regions of interaction. In some cases, very large-scale filaments are not obviously associated with any individual YSO. Maser models have been successful in predicting the association of different $\mathrm{OH}$ transitions and it is possible that small-scale anti-correlations with methanol may represent an earlier stage of cloud collapse. It now seems likely that in at least some cases, water and methanol masers trace near-Keplerian protostellar or even proto-planetary discs (as distinct from accretion discs or expanding shells). Polarization measurements of multiple transitions of $\mathrm{OH}$, water and now methanol allow the magnetic field to be traced on many scales under a range of conditions. In the early stages of YSO condensation the field is enhanced as it is wound up in the protostellar disc, but it decays as the gas around older objects expands. Water masers tracing denser post-shock or entrained material experience a higher magnetic field than pre-shock $\mathrm{OH}$ masers, also consistent with the relationship between magnetic field strength and density. Future multi-frequency analysis may allow compensation for the effects of Faraday rotation. When high-resolution interferometry of thermal emission becomes possible with the next generation of interferometers, we will be able to explain in detail how the magnetically-regulated kinematics and timescale of star formation are related to the chemistry and initial environment. This will answer some of the most interesting questions such as what determines whether low- or high-mass stars form, and under what conditions do planets condense?

Many of the species described in this review have higher-frequency transitions, at present only accessible to single dishes, which will be imaged by ALMA. This will also resolve lesser-known masers and bright molecular lines which may have some masing transitions at $\mathrm{mm}$ and sub-mm wavelengths such as $\mathrm{NH}_{3}$ and CCS[31], SiS[52] and HCN[103], leading to high-resolution studies of the chemical chronology of star formation and of reducing or carbon-rich environments. 
There is strong observational evidence that solitary evolved stars produce magnetic fields able to impose axial symmetry on their winds, but we cannot explain satisfactorily either how the magnetic field is generated, nor how it acts on the wind. It is necessary to stress, on the one hand, a few binary maser sources have been detected, often with clear discs and/or jets, but on the other hand, the majority of sources have no evidence for a dynamically significant companion and stringent upper limits on any rotation, yet they possess ordered magnetic fields and at least modest asymmetries. High-resolution cm-wave continuum and ALMA dust polarimetry may help to unravel this conundrum. SNR provide a useful precedent, where radio continuum and maser polarization have been shown to be in agreement.

The clumpy nature of mass loss from evolved stars has been directly observed; mass loss is concentrated in density-bounded water maser clouds (at least outside the dust formation zone) which are more effectively accelerated than the surrounding $\mathrm{OH}$ mainline maser gas. The water maser clouds also experience an enhanced magnetic field, possibly related to an origin in stellar convective cells. Within the next decade the combination of $e$-MERLIN, the EVLA and ALMA, alongside existing VLBI networks such as the EVN, VLBA and VERA, will reveal:

- The exact location of the central(?) star in relation to its masers;

- Perturbations of the stellar surface related to the mass loss process;

- Tracing an individual maser cloud through the $\mathrm{SiO}$ region, dust formation (determining whether the clump scale remains the same), the water maser zone and out through $\mathrm{OH} 1612-$ $\mathrm{MHz}$ masers and $\mathrm{CO}$.

This will lead to a much better understanding of the return of enriched matter to the ISM and the environment shaping $\mathrm{PNe}$ or $\mathrm{SNe}$.

\section{Acknowledgements}

I gratefully thank the many people whose material I have used, including Araya, Babkovskaia, Bains, Bartkiewicz, Cohen, Desmurs, Diamond, Edris, Etoka, Gledhill, Green, Gray, HarveySmith, Hoffman, Lekht, Hutawarakorn Kramer, Masheder, Matsuura, Mendoza-Torres, Murakawa, Pestalozzi, Szczerba, Szymczak, van Langevelde, Vlemmings, Wright and Yates. I particularly acknowledge everlasting gratitude to my former $\mathrm{PhD}$ supervisor and very good friend and colleague Jim Cohen, died 1 November 2006.

\section{References}

[1] The American Association of Variable Star Observers (AAVSO) http: / / www . aavso. org/

[2] Alakoz, A., 2006, in proceedings of The 8th European VLBI Network Symposium, Eds. A. Marecki et al., POS ( 8 thEVN) 038

[3] Alcolea, J. 2004, in European VLBI Network Symposium on New Developments in VLBI Science and Technology, 169 (arXiv:astro-ph/0412295)

[4] Arikawa, Y., Tatematsu, K., Sekimoto, Y., \& Takahashi, T. 1999, PASJ, 51, L7 
[5] Aspin, C., Sandell, G., \& Russell, A. P. G. 1994, A\&AS, 106, 165

[6] Babkovskaia, N., Poutanen, J., Richards, A. M. S., \& Szczerba, R. 2006, MNRAS, 370, 1921

[7] Bains, I., Gledhill, T. M., Yates, J. A., \& Richards, A. M. S. 2003, MNRAS, 338, 287

[8] Bains, I., Cohen, R. J., Louridas, A., Richards, A. M. S., Rosa-González, D., \& Yates, J. A. 2003, MNRAS, 342, 8

[9] Bains, I., Richards, A. M. S., Gledhill, T. M., \& Yates, J. A. 2004, MNRAS, 354, 529

[10] Bartkiewicz, A., Szymczak, M., Cohen, R. J., \& Richards, A. M. S. 2005, MNRAS, 361, 623

[11] Bartkiewicz, A., Szymczak, M., \& van Langevelde, H. J. 2005, A\&A, 442, L61

[12] Bartkiewicz, A. 2006, in proceedings of The 8th European VLBI Network Symposium, Eds. A. Marecki et al., POS ( 8 thEVN) 039

[13] Baudry, A., Menten, K. M., Walmsley, C. M., \& Wilson, T. L. 1993, A\&A, 271, 552

[14] Baudry, A., \& Menten, K. M. 1995, A\&A, 298, 905

[15] Baudry, A., \& Diamond, P. J. 1998, A\&A, 331, 697

[16] Bohigas, J., \& Tapia, M. 2003, AJ, 126, 1861

[17] Bowen, G. H. 1988, ApJ, 329, 299

[18] Brogan, C. L., Gelfand, J. D., Gaensler, B. M., Kassim, N. E., \& Lazio, T. J. W. 2006, ApJ, 639, L25

[19] Chapman, J. M., \& Cohen, R. J. 1986, MNRAS, 220, 513

[20] Chapman, J. M., \& Baan, W. 2007, Astrophysical masers and their environments IAU Symposium 242, CUP

[21] Chen, X., Shen, Z.-Q., Imai, H., \& Kamohara, R. 2006, ApJ, 640, 982

[22] Cohen, R. J. 1989, Reports of Progress in Physics, 52, 881

[23] Cohen, R. J., Caswell, J. et al., The Methanol Multi-Beam Survey http://www.jb.man.ac.uk/research/methanol/

[24] Cook, A. H. 1975, MNRAS, 171, 605

[25] Cotton, W. D., et al. 2006, A\&A, 456, 339

[26] Cragg, D. M., Sobolev, A. M., \& Godfrey, P. D. 2002, MNRAS, 331, 521

[27] Cragg, D. M., Sobolev, A. M., \& Godfrey, P. D. 2005, MNRAS, 360, 533

[28] Danchi, W. C., Bester, M., Degiacomi, C. G., Greenhill, L. J., \& Townes, C. H. 1994, AJ, 107, 1469

[29] Danchi, W. C., Green, W. H., Hale, D. D. S., McElroy, K., Monnier, J. D., Tuthill, P. G., \& Townes, C. H. 2001, ApJ, 555, 405

[30] Davis L. Jr., \& Greenstein J. L., 1951, ApJ, 305, 206

[31] De Gregorio-Monsalvo, I., Chandler, C. J., Gómez, J. F., Kuiper, T. B. H., Torrelles, J. M., \& Anglada, G. 2005, ApJ, 628, 789

[32] Deguchi, S., \& Watson, W. D. 1989, ApJ, 340, L17

[33] Deguchi, S., Matsunaga, N., \& Fukushi, H. 2005, PASJ, 57, L25

[34] Desmurs, J.-F., Baudry, A., Sivagnanam, P., \& Henkel, C. 2002, A\&A, 394, 975 
[35] Dodson, R., Ojha, R., \& Ellingsen, S. P. 2004, MNRAS, 351, 779

[36] Dorch, S. B. F. 2004, A\&A, 423, 1101

[37] Edris, K. A., Fuller, G. A., Cohen, R. J., \& Etoka, S. 2005, A\&A, 434, 213

[38] Elitzur, M., McKee, C. F., \& Hollenbach, D. J. 1991, ApJ, 367, 333

[39] Elitzur, M. 1992, Astronomical masers Kluwer Academic Publishers

[40] Elitzur, M., Hollenbach, D. J., \& McKee, C. F. 1992, ApJ, 394, 221

[41] Elitzur, M. 1996, ApJ, 457, 415

[42] Elitzur, M. 1998, ApJ, 504, 390

[43] Ellingsen, S. P., von Bibra, M. L., McCulloch, P. M., Norris, R. P., Deshpande, A. A. \& Phillips, C. J. 1996, MNRAS, 280, 378

[44] Engels, D., \& Leinert, C. 1994, A\&A, 282, 858

[45] Engels, D. 1994, A\&A, 285, 497

[46] Etoka, S., \& Le Squeren, A. M. 1997, A\&A, 321, 877

[47] Etoka, S., Błaszkiewicz, L., Szymczak, M., \& Le Squeren, A. M. 2001, A\&A, 378, 522

[48] Etoka, S., \& Diamond, P. 2004, MNRAS, 348, 34

[49] Etoka, S., Cohen, R. J., \& Gray, M. D. 2005, MNRAS, 360, 1162

[50] Figer, D. F., MacKenty, J. W., Robberto, M., Smith, K., Najarro, F., Kudritzki, R. P., \& Herrero, A. 2006, ApJ, 643, 1166

[51] Fish, V. L., \& Reid, M. J. 2006, ApJS, 164, 99

[52] Fonfría Expósito, J. P., Agúndez, M., Tercero, B., Pardo, J. R., \& Cernicharo, J. 2006, ApJ, 646, L127

[53] Frail, D. A., Goss, W. M., \& Slysh, V. I. 1994, ApJ, 424, L111

[54] Furuya, R. S., Kitamura, Y., Wootten, H. A., Claussen, M. J., \& Kawabe, R. 2001, ApJ, 559, L143

[55] Furuya, R. S., Kitamura, Y., Wootten, A., Claussen, M. J., \& Kawabe, R. 2003, ApJS, 144, 71

[56] Gallimore, J. F., Cool, R. J., Thornley, M. D., \& McMullin, J. 2003, ApJ, 586, 306

[57] Garrigue, J. P., \& Mennessier, M. O. 1980, A\&A, 81, L13

[58] Spitzer Galactic Legacy Infrared Mid-Plane Survey Extraordinaire http://www.astro.wisc.edu/glimpse/index.html

[59] Goldreich, P., Keeley, D. A., \& Kwan, J. Y. 1973, ApJ, 179, 111

[60] Gray, M. D., Field, D., \& Doel, R. C. 1992, A\&A, 262, 555

[61] Gray, M. D. 2003, MNRAS, 343, L33

[62] Guilloteau, S., Baudry, A., \& Walmsley, C. M. 1985, A\&A, 153, 179

[63] Hartquist, T. W., \& Dyson, J. E. 1997, A\&A, 319, 589

[64] Harvey-Smith, L., \& Cohen, R. J. 2005, MNRAS, 356, 637

[65] Harvey-Smith, L., \& Cohen, R. J. 2006, MNRAS, 371, 1550 
[66] Harvey-Smith, L., 2006, in proceedings of The 8th European VLBI Network Symposium, Eds. A. Marecki et al., POS ( 8 thEVN) 037

[67] Haschick, A. D., \& Ho, P. T. P. 1985, ApJ, 292, 200

[68] Herpin, F., Baudry, A., Thum, C., Morris, D., \& Wiesemeyer, H. 2006, A\&A, 450, 667

[69] Hewitt, J. W., Yusef-Zadeh, F., Wardle, M., Roberts, D. A., \& Kassim, N. E. 2006 arXiv:astro-ph/0602210

[70] Ho, P. T. P., Haschick, A. D., \& Israel, F. P. 1981, ApJ, 243, 546

[71] Hoffman, I. M., Goss, W. M., Brogan, C. L., Claussen, M. J., \& Richards, A. M. S. 2003, ApJ, 583, 272

[72] Hoffman, I. M., Goss, W. M., Palmer, P., \& Richards, A. M. S. 2003, ApJ, 598, 1061

[73] Hoffman, I. M., Goss, W. M., Brogan, C. L., \& Claussen, M. J. 2005, ApJ, 620, 257

[74] Hoffman, I. M., Goss, W. M., Brogan, C. L., \& Claussen, M. J. 2005, ApJ, 627, 803

[75] Homna, M., 2006, in proceedings of The 8th European VLBI Network Symposium, Eds. A. Marecki et al., POS ( 8thEVN) 041

[76] Hutawarakorn, B., Cohen, R. J., \& Brebner, G. C. 2002, MNRAS, 330, 349

[77] Hutawarakorn, B., \& Cohen, R. J. 2003, MNRAS, 345

[78] Kalenskï, S. V., Promyslov, V. G., Slysh, V. I., Bergman, P., \& Winnberg, A. 2006, ARep, 50, 289

[79] Kameya, O., 2006, in proceedings of The 8th European VLBI Network Symposium, Eds. A. Marecki et al., POS ( 8thEVN) 040

[80] Kirrane, T.-M. 1987, PhD Thesis, University of Manchester

[81] Kurayama, T., Sasao, T., \& Kobayashi, H. 2005, ApJ, 627, L49

[82] Lekht, E. E., \& Sorochenko, R. L. 2001, ARep, 45, 113

[83] Lekht, E. E., Mendoza-Torres, J. E., \& Berulis, I. I. 2002, ARep, 46, 57

[84] Lekht, E. E., \& Richards, A. M. S. 2003, ARep, 47, 326

[85] Lekht, E. E., Rudnitskij, G. M., Mendoza-Torres, J. E., \& Tolmachev, A. M. 2005, A\&A, 437, 127

[86] Lekht, E. E., Trinidad, M. A., Mendoza-Torres, J. E., Rudnitskij, G. M., \& Tolmachev, A. M. 2006, A\&A, 456, 145

[87] Little-Marenin, I. R. 1986, ApJ, 307, L15

[88] Lockett, P., Gauthier, E., \& Elitzur, M. 1999, ApJ, 511, 235

[89] Masheder, M. R. W., van Langevelde, H. J., Richards, A. M. S., Greenhill, L., \& Gray, M. D. 1999, NAR, 43, 563

[90] Matsunaga, N., Deguchi, S., Ita, Y., Tanabe, T., \& Nakada, Y. 2005, PASJ, 57, L1

[91] Matsuura, M., et al. 2006, ApJ, 646, L123

[92] Menten, K. M., Reid, M. J., Pratap, P., Moran, J. M., \& Wilson, T. M. 1996, ApJ, 401, L39

[93] Migenes, V., \& Reid, M. J. 2002, Cosmic masers IAU Symposium 206, ASP

[94] Milne, D. K. 1987, AuJPh, 40, 771 
[95] Minier, V., Booth, R. S., \& Conway, J. E. 1998, A\&A, 336, L5

[96] Monnier, J. D., et al. 2004, ApJ, 605, 436

[97] Moscadelli, L., Menten, K. M., Walmsley, C. M., \& Reid, M. J. 2002, ApJ, 564, 813

[98] Mouschovias, T. Ch. 1987, in Physical Processes in Interstellar Clouds, Eds. G. E. Morfill \& M. Scholer (Dordrecht: Reidel), 453

[99] Munari, U., et al., 2002, A\&A, 389, L51

[100] Murakawa, K., Yates, J. A., Richards, A. M. S., \& Cohen, R. J. 2003, MNRAS, 344, 1

[101] Nakada, Y., Izumiura, H., Onaka, T., Hashimoto, O., Ukita, N., Deguchi, S., \& Tanabe, T. 1987, ApJ, 323, L77

[102] Nakashima J.-i., \& Deguchi, S. 2006, ApJ, 647, L139

[103] Olofsson, H., Lindqvist, M., Nyman, L.-A., \& Winnberg, A. 1998, A\&A, 329, 1059

[104] Ostrowski-Fukuda, T. A., Stencel, R. E., Kemball, A., Harper, G., \& Diamond, P. J. 2002, BAAS, 34,1291

[105] Pestalozzi, M. R., Elitzur, M., Conway J. E. \& Booth, R. S. 2004, ApJ, 603, L113

[106] Pihlström, Y. 2006, in proceedings of The 8th European VLBI Network Symposium, Eds. A. Marecki et al., POS ( 8thEVN) 034

[107] Richards, A. M. S., Yates, J. A., \& Cohen, R. J. 1998, MNRAS, 299, 319

[108] Richards, A. M. S., Yates, J. A., \& Cohen, R. J. 1996, MNRAS, 282, 665

[109] Richards, A. M. S., Yates, J. A., \& Cohen, R. J. 1999, MNRAS, 306, 954

[110] Richards, A. M. S., Cohen, R. J., Bains, I., \& Yates, J. A. 1999, in IAU Symp. 191: Asymptotic Giant Branch Stars, 191, 315

[111] Richards, A. M. S., Cohen, R. J., Crocker, M., Lekht, E. E., Mendoza, E., \& Samodurov, V. A. 2005, AP\&SS, 295, 19

[112] Rodríguez, L. F., Anglada, G., Torrelles, J. M., Mendoza-Torres, J. E., Haschick, A. D., \& Ho, P. T. P. 2002, A\&A, 389, 572

[113] Rudnitskij, G. M., Lekht, E. E., Mendoza-Torres, J. E., Pashchenko, M. I., \& Berulis, I. I. 2000, A\&AS, 146, 385

[114] Shimoikura, T., Kobayashi, H., Omodaka, T., Diamond, P. J., Matveyenko, L. I., \& Fujisawa, K. 2005, ApJ, 634, 459

[115] Simis, Y. J. W., Icke, V., \& Dominik, C. 2001, A\&A, 371, 205

[116] Slysh, V. I., \& Migenes, V. 2006, MNRAS, 369, 1497

[117] Soker, N., \& Zoabi, E. 2002, MNRAS, 329, 204

[118] Szczerba, R., Szymczak, M., Babkovskaia, N., Poutanen, J., Richards, A. M. S., \& Groenewegen, M. A. T. 2006, A\&A, 452, 561

[119] Szymczak, M., \& Cohen, R. J. 1997, MNRAS, 288, 945

[120] Szymczak, M., Cohen, R. J., \& Richards, A. M. S. 1998, MNRAS, 297, 1151

[121] Szymczak, M., Cohen, R. J., \& Richards, A. M. S. 1999, MNRAS, 304, 877 
[122] Szymczak, M., Hrynek, G. \& Kus, A. J. 2000, A\&AS, 143, 269

[123] Szymczak, M., Cohen, R. J., \& Richards, A. M. S. 2001, A\&A, 371, 1012

[124] Szymczak, M., \& Gérard, E. 2004, A\&A, 414, 235

[125] Szymczak, M., 2006, in proceedings of The 8th European VLBI Network Symposium, Eds. A. Marecki et al., PoS ( 8 thEVN) 047

[126] Torrelles, J. M., et al. 2001, Nat, 411, 277

[127] Uchida Y., Shibata K., 1985, PASJ, 37, 515

[128] Vlemmings, W., Diamond, P. J., \& van Langevelde, H. J. 2001, A\&A, 375, L1

[129] Vlemmings, W. H. T., Diamond, P. J., \& van Langevelde, H. J. 2002, A\&A, 394, 589

[130] Vlemmings, W. H. T., \& van Langevelde, H. J. 2005, A\&A, 434, 1029

[131] Vlemmings, W. H. T., van Langevelde, H. J., \& Diamond, P. J. 2005, A\&A, 434, 1029

[132] Vlemmings, W. H. T. 2006, A\&A, 445, 1031

[133] Vlemmings, W. H. T., Harvey-Smith, L., \& Cohen, R. J. 2006, MNRAS, 371, L26

[134] Vlemmings, W. H. T., \& Diamond, P. J. 2006, ApJ, 648, L59

[135] Vlemmings, W. H. T., Diamond, P. J., van Langevelde, H. J., \& Torrelles, J. M. 2006, A\&A, 448, 597

[136] Watson, W. D., \& Wyld, H. W. 2001, ApJ, 558, L55

[137] Weisberg, J. M., Johnston, S., Koribalski, B., \& Stanimirović, S. 2005, Science, 309, 1

[138] Willems, F. J., \& de Jong, T. 1986, ApJ, 309, L39

[139] Wright, M. M., Gray, M. D., \& Diamond, P. J. 2004, MNRAS, 350, 1253

[140] Wright, M. M., Gray, M. D., \& Diamond, P. J. 2004, MNRAS, 350, 1272

[141] Yamamura, I., Dominik, C., de Jong, T., Waters, L. B. F. M., \& Molster, F. J. 2000, A\&A, 363, 629

[142] Yates, J. A., \& Cohen, R. J. 1994, MNRAS, 270, 958

[143] Zijlstra, A. A., Chapman, J. M., te Lintel Hekkert, P., Likkel, L., Comeron, F., Norris, R. P., Molster, F. J., \& Cohen, R. J. 2001, MNRAS, 322, 280 